\title{
Polynomial Rewritings from Expressive Description Logics with Closed Predicates to Variants of Datalog
}

\author{
Shqiponja Ahmetaj \\ TU Wien, Austria \\ Magdalena Ortiz \\ TU Wien, Austria \\ Mantas Šimkus \\ TU Wien, Austria
}

AHMETAJ@DBAI.TUWIEN.AC.AT

ORTIZ@KR.TUWIEN.AC.AT

SIMKUS@DBAI.TUWIEN.AC.AT

\begin{abstract}
In many scenarios, complete and incomplete information coexist. For this reason, the knowledge representation and database communities have long shown interest in simultaneously supporting the closed- and the open-world views when reasoning about logic theories. Here we consider the setting of querying possibly incomplete data using logic theories, formalized as the evaluation of an ontology-mediated query $(O M Q)$ that pairs a query with a theory, sometimes called an ontology, expressing background knowledge. This can be further enriched by specifying a set of closed predicates from the theory that are to be interpreted under the closed-world assumption, while the rest are interpreted with the open-world view. In this way we can retrieve more precise answers to queries by leveraging the partial completeness of the data.

The central goal of this paper is to understand the relative expressiveness of ontology-mediated query lan-

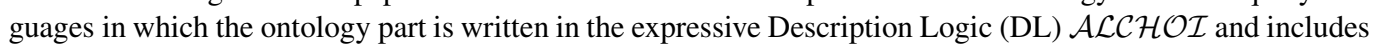
a set of closed predicates. We consider a restricted class of conjunctive queries. Our main result is to show that every query in this non-monotonic query language can be translated in polynomial time into DATALOG with negation as failure under the stable model semantics. To overcome the challenge that DATALOG has no

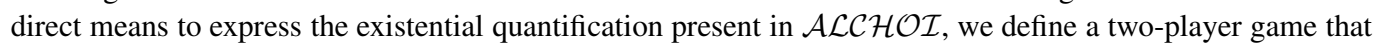
characterizes the satisfaction of the ontology, and design a DATALOG query that can decide the existence of a winning strategy for the game. If there are no closed predicates-in the case of querying an $\mathcal{A L C H O \mathcal { I }}$ knowledge base-our translation yields a positive disjunctive DATALOG program of polynomial size. To the best of our knowledge, unlike previous translations for related fragments with expressive (non-Horn) DLs, these are the first polynomial time translations.
\end{abstract}

\section{Introduction}

Ontology-mediated queries (OMQs) are an extension of standard database query languages, which allow to obtain more answers from incomplete data by using domain knowledge given as an ontology. In a nutshell, an ontology is a logical theory describing a domain of interest. It explicates the entities that are relevant in the domain, their properties, and the relations between them, in a formal logical language with welldefined syntax. The most popular languages for writing ontologies are based on Description Logics (DLs), a prominent family of decidable fragments of first-order logic [4], and on related rule languages like DATALOG \pm [13]. Advocated mostly as a tool to provide users and applications a common understanding of the domain of discourse, ontologies are applied in a diversity of areas, including the Web, life sciences, and many others.

A successful use case for ontologies is in data management, where the ontology-based data access $(O B D A)$ paradigm advocates the use of ontologies as conceptual views of possibly incomplete and heterogeneous data sources, facilitating their access. OMQs were proposed in this setting. In a nutshell, an OMQ pairs a database query and an ontology, and the latter is used to infer better answers from incomplete data. For example, the following axiom expresses that Bachelor students are students. It is written in DL syntax, as a so-called TBox $\mathcal{T}_{1}$ :

$$
\text { BScStud } \sqsubseteq \text { Student }
$$


Consider an OMQ $\left(\mathcal{T}_{1}, q_{1}\right)$ that pairs $\mathcal{T}_{1}$ and a (unary) instance query $q_{1}(x)=\operatorname{Student}(x)$ that retrieves all students. If we pose this OMQ over a database that stores that Alice is a Bachelor student and Bob a student; in our case, written as a DL $A B o x$ with two assertions $\{\operatorname{BScStud}(a)$, Student $(b)\}$, then we obtain both $a$ (Alice) and $b$ (Bob) as (certain) answers. Note that $q_{1}$ alone, without the knowledge in $\mathcal{T}_{1}$, would only retrieve $b$ as an answer. OMQs have received extensive attention over the last decade, and are still motivating major research efforts in the database and knowledge representation research communities; see, for example, the following works and their references [12, 10, 30].

The open-world semantics is the central property that makes OMQs suitable for incomplete data sources. The facts in the database only describe the world partially; other facts that are not present may also be true. Here Alice is a student, although this is not present in the data. However, viewing all data as incomplete can result in too few certain answers. For example, we let the TBox $\mathcal{T}$ contain $\mathcal{T}_{1}$ and the following axioms, stating that every student attends some course, and that bachelor students cannot attend graduate courses:

$$
\text { Student } \sqsubseteq \text { Jattends. Course } \quad \text { BScStud } \sqsubseteq \forall \text { attends. } \neg \text { GradCourse }
$$

Now we take the following $\operatorname{ABox} \mathcal{A}$ :

$$
\left\{\operatorname{BScStud}(a), \quad \operatorname{Course}\left(c_{1}\right), \quad \text { Course }\left(c_{2}\right), \quad \operatorname{GradCourse}\left(c_{2}\right)\right\}
$$

There are no certain answers for the OMQ $\left(\mathcal{T}, q_{2}\right)$ with $q_{2}(x, y)=$ attends $(x, y)$; intuitively, we do not know which course Alice attends. However, if $c_{1}$ and $c_{2}$ are known to be the only courses, then $\left(a, c_{1}\right)$ should become a certain answer, since Alice must attend some course and it cannot be $c_{2}$.

Reasoning in the presence of partial completeness has always been considered a very relevant but challenging problem, both in the databases and in the knowledge representation communities. The last few years have seen a renewed interest in the topic. For a survey of many works on databases combining complete and incomplete information, the reader may refer to [45] and its references. In DLs, closed predicates have been advocated as a natural way to combine complete and incomplete knowledge. Here a set of predicates is explicitly declared as closed; these are assumed complete and given a closed-world semantics [24, 38]. In our example above, $\left(a, c_{1}\right)$ is a certain answer if we declare Course as closed predicate. Note that closed predicates make queries non-monotonic: although $\left(a, c_{1}\right)$ is a certain answer over $\mathcal{A}$, it is not a certain answer over the extended set of facts $\mathcal{A}^{\prime}=\mathcal{A} \cup\left\{\right.$ Course $\left.\left(c_{3}\right)\right\}$.

The main goal of this paper is to investigate the relative expressiveness of OMQ languages where the ontology is written in an expressive DL with closed predicates, in terms of more traditional query languages like DATALOG. More precisely, we are interested in the following problem: given an OMQ $Q$, is it possible to obtain a query $Q^{\prime}$ in a suitable fragment of DATALOG such that, for any $\operatorname{ABox} \mathcal{A}$, the certain answers to $Q$ and $Q^{\prime}$ coincide, and the size of $Q^{\prime}$ is bounded by a polynomial in $Q$ ? Such rewritings are central in OMQ research. The existence of $Q^{\prime}$ and its size are crucial for understanding the expressive power and succinctness of different families of OMQs. For some less expressive DLs, rewritings have also paved the way to scalable OMQ answering by reusing existing technologies. As we discuss below, finding such rewritings has been a very active research field and produced many results. However, the vast majority of these results are for OMQs whose ontological component is in a so-called lightweight $D L$, and use as target languages either firstorder queries, or fragments of standard (positive) DATALOG, which have as common feature polynomial data

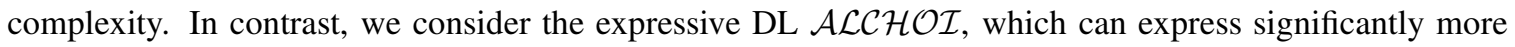
complex structures of knowledge, but as it is coNP-hard in data complexity [47], a rewriting into plain (nondisjunctive) DATALOG cannot exist under standard computational complexity assumptions. Moreover, for DLs that are not polynomial in combined complexity, most rewritings need exponential time and generate an exponentially larger DATALOG program. Last but not least, the non-monotonicity caused by closed predicates means that our OMQs cannot be rewritten into monotonic variants of DATALOG, like positive DATALOG (with or without disjunction).

The OMQs in this paper take the form $(\mathcal{T}, \Sigma, \mathfrak{q})$, where $\mathcal{T}$ a TBox in the very expressive DL $\mathcal{A L C H O \mathcal { I }}$ and $\Sigma$ are the closed predicates. For the query language of $\mathfrak{q}$, a desirable candidate is the class of conjunctive queries (CQs), a popular database query language and the language of choice in the OMQ literature. 
Unfortunately, allowing CQs and closed predicates precludes the existence of a polynomial sized $Q^{\prime}$ under standard complexity assumptions, even if we restrict the ontology language. Indeed, in the presence of closed predicates, OMQ answering with CQs is 2EXPTIME-hard already for the very restricted fragments of $\mathcal{A L C H O \mathcal { I } \text { known as } \mathcal { E L } \text { and DL-Lite }} \mathcal{R}_{\mathcal{R}}$ [42], while entailment in DATALOG with negation is in coNEXPTIME $^{\mathrm{NP}}$. Therefore in our OMQs, $\mathfrak{q}$ is from a restricted class of CQs that we call $c$-acyclic, and that generalizes instance queries and acyclic conjunctive queries. We propose a polynomial time translation of any given OMQ $Q=(\mathcal{T}, \Sigma, \mathfrak{q})$ as above, into a DATALOG program extended with negation under the stable model semantics.

More precisely, the paper presents two main contributions:

- We first provide a game-theoretic characterization of the semantics of our OMQs. To overcome the challenge that DATALOG has no direct means to express the existential quantification present in $\mathcal{A L C H O I}$, we define a two-player game that, in a nutshell, verifies whether a given structure (essentially, an input $\mathrm{ABox} \mathcal{A}$ possibly extended with additional facts about the same individuals) can be extended into a model of the given ontology, while preserving the (non)entailment of ground facts.

- We construct, for a given c-acyclic OMQ $Q=(\mathcal{A L C H O \mathcal { I }}, \Sigma, \mathfrak{q})$ a query $\left(P_{Q}, q\right)$, where $P_{Q}$ is a DATALOG program with negation, which verifies the existence of a winning strategy for the game above. We prove that the certain answers of $Q$ and $\left(P_{Q}, q\right)$ coincide over every input ABox $\mathcal{A}$ over the concept and role names occurring in $\mathcal{T}$. $P_{Q}$ does not depend on the input data, and crucially, its size is polynomial. The rules in $P_{Q}$ are non-ground, and use predicates whose arities depend on the input $\mathcal{T}$. To our knowledge, this is the first polynomial time translation of an expressive (non-Horn) DL into a variant of DATALOG.

With these constructions in place, we can also obtain the following results:

- We obtain optimal complexity bounds, for deciding whether a tuple $\vec{a}$ is a certain answer of $(\mathcal{T}, \Sigma, \mathfrak{q})$ over a input $\mathrm{ABox} \mathcal{A}$, namely coNP-completeness in data complexity and EXPTiME-completeness in combined complexity. The upper bound for data complexity follows from the complexity of reasoning in DATALOG with negation. For the upper bound on combined complexity, we carefully analyze the shape of the program $P$ that results from our rewriting, and we show that the resulting rules fall into a suitably restricted fragment. Note that entailment from unrestricted DATALOG programs with negation is coNEXPTIME-complete [19].

- We study the case when closed predicates are absent. We show that the considered OMQs can be translated into a positive disjunctive DATALOG program that uses, and inherently needs, the built-in inequality predicate $\neq$. If in addition nominals are disallowed from ontologies, then the inequality predicate is not used.

- Our polynomial time translations heavily rely on a pair of constants, intuitively corresponding to the bits 0 and 1, that are introduced by rules of the target program, i.e., our target variants of DATALOG support constants in general rules. We argue that disallowing this leads to the non-existence of polynomial time translations, under the usual assumptions in complexity theory.

A preliminary version of this article has been published in [1], and a simplified version of this translation for

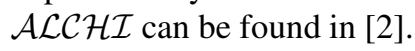

\section{Related Work}

Combining complete and incomplete information In the database community, there is considerable amount of work on viewing the data as a mixture of complete and incomplete information. For example, in Master Data Management [23], often some relations in an organization's database can be considered complete (e.g., the table of company's products), while the remaining ones may miss tuples, i.e. may be incomplete (e.g., the customers' phone numbers). The question of whether a database with complete and 
incomplete predicates has complete information to answer a query has received significant attention [23, 18]; these works focus on the complexity for variations of the problem when inclusion dependencies are given as database constraints.

When reasoning about privacy, some or all tuples in selected database relations may be hidden for privacy reasons, and thus from the users' viewpoint such relations are incomplete [8, 9]. This work, quite related to ours, focuses on visible and invisible tables. The visible tables are database predicates that are assumed complete, while the invisible tables are predicates that should be seen as incomplete. Among others, the authors consider the Positive Query Implication problem for guarded tuple-generating dependencies (TGDs) (also known as existential rules) and guarded disjunctive TGDs, which matches the usual query answering problem for DLs in the presence of closed predicates, the task we consider in this paper. The DL $\mathcal{A L C H O I}$ can be seen as an ontology language that is orthogonal to guarded disjunctive TGDs, which support predicates of arbitrary arity. However, unlike us, they focus on the complexity of answering variations of conjunctive queries, showing that query answering is already EXPTIME-complete in data complexity, and 2EXPTIMEcomplete in combined complexity. We consider only a restricted class of conjunctive queries.

In the DL setting, closed predicates were introduced in [49]. In that work, ABoxes are replaced by DBoxes, which are syntactically the same, but interpreted by taking all predicates occurring in the ABox as closed. It was shown there that this change results in coNP hard instance checking, even for lightweight DLs. The authors also prove that in expressive DLs with nominals DBoxes can be expressed rather naturally as part of the TBox. We remark that this encoding is only for a given DBox (i.e., dataset), and hence it is not useful for obtaining data-independent rewritings of OMQs with closed predicates. More recent works on OMQs with closed predicates have focused on the complexity of their evaluation, e.g., [42, 38, 24].

Another research direction that brings some form of closed-word reasoning to DLs is the so-called hybrid knowledge bases that combine DL ontologies with DATALOG rules (see, e.g., [36, 46, 20, 40]). Hybrid knowledge bases usually consist of a $\operatorname{logic}$ program $\mathcal{P}$ and a TBox $\mathcal{T}$ in some DL. Typically, the signature is partitioned into DL and rule predicates, and the closed world assumption is applied to the latter, but not to the former. Most hybrid formalisms impose syntactic safety conditions, necessary for reasoning to be decidable. Some of these formalisms allow for default negation, and can thus be seen as extensions of DLs with non-monotonic reasoning. However, most hybrid languages cannot naturally simulate the full effect of closed predicates in DLs. A relevant exception is the recently introduced language of Clopen knowledge bases [5] that combines non-monotonic disjunctive DATALOG rules under the stable model semantics with DL ontologies, as well as open and closed predicates. For reasoning about such $\mathrm{KB}$, an algorithm that rewrites a given KB into non-monotonic disjunctive DATALOG was proposed and implemented. However, the rewriting algorithm is only for a fragment that significantly restricts the use of closed predicates, and results in a program of exponential size.

Query rewriting In the context of DLs, rewriting OMQs into standard query languages, such as firstorder (FO) queries (SQL, non-recursive DATALOG) or DATALOG, is considered one of the most prominent approaches for OMQ answering. FO-rewritings for members of the $D L$-Lite family were originally proposed in [14, 15], but those rewritings take exponential time. For $D L-L_{i t e} \mathcal{R}_{\mathcal{R}}$, the authors in [26] propose a rewriting into a polynomially-sized non-recursive DATALOG program, assuming that the data contains some fixed number of constants. It was then shown in [27] that without the additional assumption on the fixed number of constants polynomial FO-rewritings for OMQs consisting of DL-Lite $\mathcal{R}_{\mathcal{R}}$ and (U)CQs cannot exist. The rewritings presented in this paper also use a few constants, which are supported by the DATALOG variants we employ as target query languages. In [35], the authors introduced the combined approach as a means to obtain FO-rewritings for languages, like $\mathcal{E} \mathcal{L}$, that are more expressive than DL-Lite. The central idea is to, additionally to query rewriting, modify the ABox by incorporating inferences from the TBox. Note that, in this case, the resulting rewriting is computed specifically for the given ABox; in contrast, our rewriting is independent of a particular ABox.

In the presence of closed predicates, the only rewritability results are FO-rewritability for the core fragment of DL-Lite [39], and a rewriting algorithm for queries that satisfy some strong definability criteria [49]. Since closed predicates cause coNP-hardness in data complexity already for instance queries in many 
lightweight DLs, the existence of FO-rewritings is ruled out. The recent rewriting for Clopen knowledge bases [5], as mentioned, imposes restrictions on the use of closed predicates, and results in a program of exponential size.

Many DLs are not FO-rewritable, but can be rewritten into monotonic DATALOG queries, leading to implemented systems, e.g., [44, 21, 51]. The pioneering work in [33] showed that instance queries in an expressive extension of $\mathcal{A L C}$ can be rewritten into a program in disjunctive DATALOG, using a constant number of variables per rule, but exponentially many rules. The first translation from conjunctive queries $(C Q s)$ in expressive DLs without closed predicates $(\mathcal{S H}, \mathcal{S H} \mathcal{Q})$ to programs in disjunctive DATALOG was introduced in [22], but the program may contain double exponentially many predicates. For $\mathcal{A L C}$ and for union of CQs, the existence of exponential rewritings into disjunctive DATALOG was shown recently [12], and for restricted fragments of $\mathcal{S H I}$ and classes of CQs translations to DATALOG were investigated in [34]. A polynomial time DATALOG translation of instance queries was proposed in [43], but for a so-called Horn$D L$ that lacks disjunction. To our knowledge, this was until now the only polynomial rewriting for a DL that is not FO-rewritable.

There is also a noticeable amount of works on query rewritings for variants of guarded TGDs. For instance, it was shown in [6, 29] that OMQs, where the query is given as a (union of) conjunctive queries and the ontology as a set of guarded TGDs or more general classes of dependencies, can be rewritten into a plain DATALOG program. The authors in [12] propose a rewriting into a disjunctive DATALOG program for guarded disjunctive TGDs and a union of conjunctive queries. However, the rewritings mentioned in [6, 29, 12] take exponential time, even if the number of variables in each (disjunctive) TGD is bounded by a constant. For guarded TGDs with a bound on the size of the schema and for linear TGDs, the authors in [28, 30] propose rewritings into polynomially-sized non-recursive DATALOG programs. We note that, for linear TGDs of bounded arity, this result follows from [26, 27].

Adapting the techniques presented in this paper, we showed in [3] that instance queries mediated by ontologies specified as guarded disjunctive TGDs without closed predicates can be rewritten into disjunctive DATALOG. The rewriting is polynomial if the number of variables in each TGD is bounded; we note that the TGDs considered in that work do not allow for constants (essentially, nominals). Although the latter rewriting is inspired by the techniques presented in this paper, the higher arities and the rather relaxed syntax of guarded disjunctive TGDs makes the adaptation highly non-trivial. In the absence of disjunction, that is for non-disjunctive TGDs, we additionally propose a rewriting into a (plain) DATALOG program; similarly as above, it is polynomial if the number of variables per TGD is bounded. We remark that the data complexity of such OMQs in the presence of closed predicates has been shown to be PSPACE-hard [7]. Therefore, under common assumptions in complexity theory, our rewritings cannot be generalized to such OMQs with closed predicates.

\section{Organization}

This paper is organized as follows. In Section 2 we introduce the DL $\mathcal{A L C H O I}$ and the normal form we consider in this paper. We then define in Section 3 the (ontology-mediated) query answering problem for $\mathcal{A L C H O I}$ knowledge bases in the presence of closed predicates. Section 4 is devoted to the game-like characterization of models, and in particular, we tie the inclusion of a tuple in a certain answer of a given OMQ over an input ABox to the existence of a winning strategy in a two-player game. The existence of such a strategy can be decided by a type-marking algorithm, which is presented in Section 5. In Section 6, we first introduce the required variants of DATALOG, and give an implementation of the marking algorithm from Section 5 as a DATALOG program with negation under the stable model semantics. We proceed by carefully analyzing the complexity of the program that results from our rewriting. We also consider the restricted case when closed predicates, and possibly nominals, are absent from OMQs. At the end of the section, we discuss the important role that constants are playing in our translations. Conclusions and directions for future work are given in Section 7. For better readability, a part of a rather long and technical proof is given in the Appendix 8 


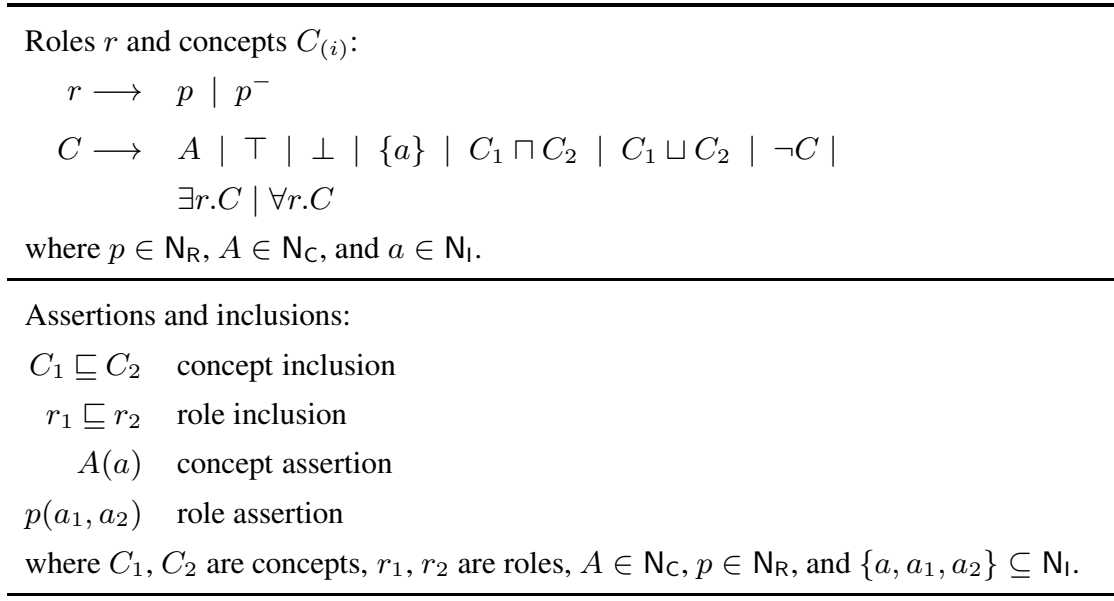

Table 1: Syntax of $\mathcal{A L C H O I}$

\section{DL Preliminaries}

In this section we define the Description Logics and OMQ languages we use in this paper.

\section{$\mathcal{A L C H O I}$ with Closed Predicates}

Syntax We recall the standard definition of the DL $\mathcal{A L C H O I}$ summarized in Table 1 . We assume countably infinite, mutually disjoint sets $\mathrm{N}_{\mathrm{R}}$ of role names, $\mathrm{N}_{\mathrm{C}}$ of concept names, and $\mathrm{N}_{\mathrm{I}}$ of individual names to build complex concepts and roles. Intuitively, concepts are unary relations used to describe classes of objects and roles are binary relations used to describe relations between objects. These expressions are then used in inclusions and assertions. The former express general dependencies to be satisfied by data instances, while the latter assert the membership of specific (pairs of) individuals in concepts and roles. A (plain) knowledge base $(K B)$ is a tuple $\mathcal{K}=(\mathcal{T}, \mathcal{A})$, where $\mathcal{T}$ is a finite set of inclusions called a $T B o x$, and $\mathcal{A}$ is a finite set of assertions called an ABox. A $K B$ with closed predicates is a triple $\mathcal{K}=(\mathcal{T}, \Sigma, \mathcal{A})$, where $(\mathcal{T}, \mathcal{A})$ is a plain $\mathrm{KB}$ and $\Sigma \subseteq \mathrm{N}_{\mathrm{C}} \cup \mathrm{N}_{\mathrm{R}}$ is the set of closed predicates in $\mathcal{K}$.

Semantics We recall the usual semantics of DL KBs, defined in terms of interpretations, relational structures over a (possibly infinite) non-empty domain and a signature consisting of unary predicates (the concept names), binary predicates (the role names), and constants (the individuals).

Definition 1. An interpretation is a pair $\mathcal{I}=\left\langle\Delta^{\mathcal{I}},{ }^{\mathcal{I}}\right\rangle$ where $\Delta^{\mathcal{I}} \neq \emptyset$ is the domain, and ${ }^{\mathcal{I}}$ is an interpretation function with $A^{\mathcal{I}} \subseteq \Delta^{\mathcal{I}}$ for each $A \in \mathrm{N}_{\mathrm{C}}, p^{\mathcal{I}} \subseteq \Delta^{\mathcal{I}} \times \Delta^{\mathcal{I}}$ for each $p \in \mathrm{N}_{\mathrm{R}}$, and $a^{\mathcal{I}} \in \Delta^{\mathcal{I}}$ for each $a \in \mathrm{N}_{1}$. The function ${ }^{\mathcal{I}}$ is extended to all $\mathcal{A L C H O I}$ concepts and roles as usual, see Table 2

Consider an interpretation $\mathcal{I}$. For an inclusion $q_{1} \sqsubseteq q_{2}$, we say that $\mathcal{I}$ satisfies $q_{1} \sqsubseteq q_{2}$ and write $\mathcal{I} \models q_{1} \sqsubseteq q_{2}$ if $q_{1}^{\mathcal{I}} \subseteq q_{2}^{\mathcal{I}}$. For an assertion $\beta$ of the form $q(\vec{a})$, we say that $\mathcal{I}$ satisfies $q(\vec{a})$, in symbols $\mathcal{I} \models q(\vec{a})$, if $(\vec{a})^{\mathcal{I}} \in q^{\mathcal{I}}$. For $\Gamma$ a TBox or ABox, we write $\mathcal{I} \models \Gamma$ if $\mathcal{I} \models \alpha$ for all $\alpha \in \Gamma$. The notion of satisfaction extends naturally to plain $\mathrm{KBs}: \mathcal{I} \models(\mathcal{T}, \mathcal{A})$ if $\mathcal{I} \models \mathcal{T}$ and $\mathcal{I} \models \mathcal{A}$. For KBs with closed predicates, we need the following notion. Let $\mathcal{A}$ be an $\mathrm{ABox}$ and $\Sigma \subseteq \mathrm{N}_{\mathrm{C}} \cup \mathrm{N}_{\mathrm{R}}$. We write $\mathcal{I} \models_{\Sigma} \mathcal{A}$ if:

(a) $\mathcal{I} \models \mathcal{A}$,

(b) for all $A \in \Sigma \cap \mathrm{N}_{\mathrm{C}}$, if $e \in A^{\mathcal{I}}$, then $A(e) \in \mathcal{A}$, and

(c) for all $r \in \Sigma \cap \mathrm{N}_{\mathrm{R}}$, if $\left(e_{1}, e_{2}\right) \in r^{\mathcal{I}}$, then $r\left(e_{1}, e_{2}\right) \in \mathcal{A}$.

Then, for a $\mathrm{KB} \mathcal{K}=(\mathcal{T}, \Sigma, \mathcal{A})$, we write $\mathcal{I} \models \mathcal{K}$ if the following hold:

(i) $a \in \Delta^{\mathcal{I}}$ and $a^{\mathcal{I}}=a$ for each $a \in \mathrm{N}_{\mathrm{I}}$ occurring in $\mathcal{K}$,

(ii) $\mathcal{I} \models \mathcal{T}$, and 


$$
\begin{aligned}
& \text { Concept constructors in } \mathcal{A L C H O \mathcal { I }}: \\
&{\top^{\mathcal{I}}}=\Delta^{\mathcal{I}} \\
& \perp^{\mathcal{I}}=\emptyset \\
&\{a\}^{\mathcal{I}}=a^{\mathcal{I}} \\
&\left(\neg C_{1}\right)^{\mathcal{I}}=\Delta^{\mathcal{I}} \backslash C_{1}^{\mathcal{I}} \\
&\left(C_{1} \sqcap C_{2}\right)^{\mathcal{I}}=C_{1}^{\mathcal{I}} \cap C_{2}^{\mathcal{I}} \\
&\left(C_{1} \sqcup C_{2}\right)^{\mathcal{I}}=C_{1}^{\mathcal{I}} \cup C_{2}^{\mathcal{I}} \\
&(\exists r . C)^{\mathcal{I}}=\left\{e_{1} \mid \text { for some } e_{2} \in \Delta^{\mathcal{I}},\left(e_{1}, e_{2}\right) \in r^{\mathcal{I}} \text { and } e_{2} \in C^{\mathcal{I}}\right\} \\
&(\forall r . C)^{\mathcal{I}}=\left\{e_{1} \mid \text { for all } e_{2} \in \Delta^{\mathcal{I}},\left(e_{1}, e_{2}\right) \in r^{\mathcal{I}} \text { implies } e_{2} \in C^{\mathcal{I}}\right\} \\
& \hline
\end{aligned}
$$

Role constructors in $\mathcal{A L C H O I}$ :

$$
\left(r^{-}\right)^{\mathcal{I}}=\left\{\left(e_{1}, e_{2}\right) \mid\left(e_{2}, e_{1}\right) \in r^{\mathcal{I}}\right\}
$$

Table 2: Semantics of $\mathcal{A L C H O I}$ concepts and roles

(iii) $\mathcal{I} \models_{\Sigma} \mathcal{A}$.

Note that we make the standard name assumption (SNA) for the individuals occurring in $\mathcal{K}$ (condition (i)), which is common for closed predicates. For $\Gamma$ a plain $\mathrm{KB}$ or a $\mathrm{KB}$ with closed predicates, or a TBox $\mathcal{T}, \Gamma$ is consistent if there is some $\mathcal{I}$ that satisfies $\Gamma$. For a TBox $\mathcal{T}$ and an inclusion $q_{1} \sqsubseteq q_{2}$, we write $\mathcal{T} \models q_{1} \sqsubseteq q_{2}$, if for every interpretation $\mathcal{I}, \mathcal{I} \models \mathcal{T}$ implies $\mathcal{I} \models q_{1} \sqsubseteq q_{2}$. In what follows, if no confusion arises, we may simply say KB to refer to a KB with closed predicates.

We let $\mathrm{N}_{\mathrm{C}}^{+}=\mathrm{N}_{\mathrm{C}} \cup\left\{\{a\} \mid a \in \mathrm{N}_{\mathrm{I}}\right\} \cup\{\top, \perp\}$ be the basic concepts and the roles $r$ of the form $p, p^{-}$with $p \in \mathrm{N}_{\mathrm{R}}$ be the basic roles. For $\Gamma$ a TBox, ABox, or KB, we denote by $\mathrm{N}_{\mathrm{I}}(\Gamma), \mathrm{N}_{\mathrm{R}}(\Gamma), \mathrm{N}_{\mathrm{C}}(\Gamma), \mathrm{N}_{\mathrm{C}}^{+}(\Gamma)$, and $\mathrm{N}_{\mathrm{C}}^{+}(\mathcal{T})$, the set of individuals, role names, concept names, and basic concepts that occur in $\Gamma$, respectively. We write $r^{-}$to mean $p^{-}$if $r=p$ is a role name, and $p$ if $r=p^{-}$is the inverse of a role name. We also write $r^{(-)} \in \Sigma$ to mean $r \in \Sigma$ or $r^{-} \in \Sigma$.

As usual, we use $\sqsubseteq_{\mathcal{T}}^{*}$ to denote the smallest relation that satisfies: (1) $r \sqsubseteq_{\mathcal{T}}^{*} r$ for every basic role $r$ in $\mathcal{T}$, (2) if $r \sqsubseteq_{\mathcal{T}}^{*} s$, then $r^{-} \sqsubseteq_{\mathcal{T}}^{*} s^{-}$, (3) if $r \sqsubseteq_{\mathcal{T}}^{*} r_{1}$ and $r_{1} \sqsubseteq s \in \mathcal{T}$, then $r \sqsubseteq_{\mathcal{T}}^{*} s$.

\section{Normal Form}

Our results apply to arbitrary TBoxes, but to simplify presentation, we consider TBoxes with a restricted syntactic structure:

Definition 2. A TBox $\mathcal{T}$ is in normal form if each inclusion in $\mathcal{T}$ has one of the following forms:

$$
\begin{array}{ll}
\text { (N1) } B_{1} \sqcap \cdots \sqcap B_{n} \sqsubseteq B_{n+1} \sqcup \cdots \sqcup B_{k} & \\
\text { (N2) } A \sqsubseteq \exists r . A^{\prime} & \text { (N3) } A \sqsubseteq \forall r . A^{\prime} \quad \text { (N4) } r \sqsubseteq s
\end{array}
$$

where $r$ and $s$ are basic roles, $\left\{B_{1}, \ldots, B_{k}\right\} \subseteq \mathrm{N}_{\mathrm{C}}^{+}$, and $\left\{A, A^{\prime}\right\} \subseteq \mathrm{N}_{\mathrm{C}}$.

The inclusions of type (N2) are often called existential inclusions in this paper. It is a standard result that, in plain KBs, the TBox can be transformed into this normal form in polynomial time, while preserving the answers to any query that uses symbols from the input KB only. This does not change in the presence of closed predicates.

Proposition 1. Consider a $K B$ with closed predicates $\mathcal{K}=(\mathcal{T}, \Sigma, \mathcal{A})$. Then $\mathcal{T}$ can be transformed in polynomial time into a TBox $\hat{\mathcal{T}}$ in normal form in such a way that, for every interpretation $\mathcal{I}$, the following hold: 
(1) If $\mathcal{I} \models(\hat{\mathcal{T}}, \Sigma, \mathcal{A})$, then $\mathcal{I} \models(\mathcal{T}, \Sigma, \mathcal{A})$.

(2) If $\mathcal{I} \models(\mathcal{T}, \Sigma, \mathcal{A})$, then $\mathcal{I}$ can be extended into $\mathcal{I}^{\prime}$ such that

(a) $\mathcal{I}^{\prime} \models(\hat{\mathcal{T}}, \Sigma, \mathcal{A})$, and

(b) $q^{\mathcal{I}}=q^{\mathcal{I}^{\prime}}$ for all symbols $q$ in $\mathrm{N}_{\mathrm{I}}(\mathcal{K}) \cup \mathrm{N}_{\mathrm{R}}(\mathcal{K}) \cup \mathrm{N}_{\mathrm{C}}(\mathcal{K})$.

Proof (Sketch). The proof relies on the availability of fresh concept and role names, which are introduced in the place of complex expressions. One can show the following:

(i) Every model of $\hat{\mathcal{T}}$ is a model of $\mathcal{T}$.

(ii) If $\mathcal{I}$ is a model of $\mathcal{T}$, we can obtain an $\mathcal{I}^{\prime}$ with $\mathcal{I}^{\prime} \models \hat{\mathcal{T}}$ by suitably interpreting the fresh concept names, while preserving $q^{\mathcal{I}}=q^{\mathcal{I}^{\prime}}$ for all symbols $q$ in $\mathrm{N}_{\mathrm{I}}(\mathcal{K}) \cup \mathrm{N}_{\mathrm{R}}(\mathcal{K}) \cup \mathrm{N}_{\mathrm{C}}(\mathcal{K})$.

We omit the detailed proof of (i) and (ii), since it is standard (e.g., see the proof for $\mathcal{A L C H \mathcal { I }}$ in [50], accommodating nominals is straightforward) 11 Now (1) follows from (i). For (2), since the interpretation in $\mathcal{I}^{\prime}$ of individuals and of all predicates in $\mathcal{A}$ and $\Sigma$ is the same as in $\mathcal{I}$, it follows that $\mathcal{I} \models_{\Sigma} \mathcal{A}$ implies $\mathcal{I}^{\prime} \models_{\Sigma} \mathcal{A}$. From the latter and from $\mathcal{I}^{\prime} \models \hat{\mathcal{T}}$ we infer (a), while (b) is a consequence of the fact that $\mathcal{I}$ and $\mathcal{I}^{\prime}$ only differ in the freshly introduced predicates.

\section{Ontology-Mediated Queries}

We are now ready to introduce the query languages that are the object of our study. In this paper we consider OMQs of the form $Q=(\mathcal{T}, \Sigma, \mathfrak{q})$, where $\mathcal{T}$ is a TBox and $\Sigma \subseteq \mathrm{N}_{\mathrm{C}} \cup \mathrm{N}_{\mathrm{R}}$ is a set of closed predicates. A natural candidate language for $\mathfrak{q}$ is the language of conjunctive queries $(C Q s)$, which are essentially first-order formulas that use only existential quantification and conjunction.

Definition 3. Let $\mathrm{N}_{\mathrm{V}}$ be a countably infinite set of variables disjoint from $\mathrm{N}_{\mathrm{C}}, \mathrm{N}_{\mathrm{R}}, \mathrm{N}_{1}$. $\mathrm{A}(D L)$ atom $\alpha$ is an expression of the form $A\left(x_{1}\right)$ or $r\left(x_{1}, x_{2}\right)$ with $A \in \mathrm{N}_{\mathrm{C}}, r \in \mathrm{N}_{\mathrm{R}}$ and $\left\{x_{1}, x_{2}\right\} \subseteq \mathrm{N}_{\mathrm{V}}$. By vars $(\Gamma)$ we denote the variables occurring in a set $\Gamma$ of atoms. If no confusion arises, a tuple of variables may be identified with the set of its elements.

A conjunctive query $\mathfrak{q}$ is an expression of the form

$$
\exists \vec{y} \cdot \alpha_{1} \wedge \cdots \wedge \alpha_{n}
$$

where each $\alpha_{i}$ is an atom and $\vec{y} \subseteq \operatorname{vars}\left(\left\{\alpha_{1}, \cdots, \alpha_{n}\right\}\right)$. We may treat a CQ as a set of atoms. The variables in $\vec{x}=\operatorname{vars}\left(\left\{\alpha_{1}, \cdots \alpha_{n}\right\}\right) \backslash \vec{y}$ are called answer variables, and the arity of $\mathfrak{q}$ is defined as the arity of $\vec{x}$. We may write $\mathfrak{q}(\vec{x})$ for a CQ with answer variables $\vec{x}$. If there are no answer variables, then $\mathfrak{q}()$ is a Boolean $C Q$. If $\mathfrak{q}(\vec{x})$ consists of only one atom $\alpha$ and $\vec{x}=\operatorname{vars}(\{\alpha\})$, that is, there are no existentially quantified variables, then $\mathfrak{q}(\vec{x})$ is an instance query.

Let $\mathfrak{q}(\vec{x})$ be a CQ and $\mathcal{I}$ an interpretation. For a mapping $\pi: \operatorname{vars}(\mathfrak{q}) \rightarrow \Delta^{\mathcal{I}}$, we write $\mathcal{I} \models \pi(\mathfrak{q}(\vec{a}))$ if $\pi(\vec{x})=\vec{a}$ and $\mathcal{I} \models \pi(\alpha)$ for each atom $\alpha$ in $\mathfrak{q}$ (where we slightly abuse notation and apply $\pi$ to tuples of variables and atoms). If $\mathcal{I} \models \pi(\mathfrak{q}(\vec{a}))$ for some $\pi$, we write $\mathcal{I} \models \mathfrak{q}(\vec{a})$ and say that $\mathcal{I}$ satisfies $\mathfrak{q}(\vec{a})$. For $\mathcal{K}$ a plain $\mathrm{KB}$, or a $\mathrm{KB}$ with closed predicates and for a tuple of constants $\vec{a}$, we say $\mathcal{K}$ entails $\mathfrak{q}(\vec{a})$, written $\mathcal{K} \models \mathfrak{q}(\vec{a})$, if $\mathcal{I} \models \mathfrak{q}(\vec{a})$ for every $\mathcal{I}$ such that $\mathcal{I} \models \mathcal{K}$.

Let $Q=(\mathcal{T}, \Sigma, \mathfrak{q}(\vec{x}))$ be an OMQ, where $\mathfrak{q}(\vec{x})$ is a CQ of arity $n$. For an ABox $\mathcal{A}$ and $\vec{a} \in \mathrm{N}_{1}^{n}, \vec{a}$ is a certain answer to $Q$ over $\mathcal{A}$ if $(\mathcal{T}, \Sigma, \mathcal{A}) \models \mathfrak{q}(\vec{a})$. We denote with $\operatorname{cert}(Q, \mathcal{A})$ the set of certain answers of $Q$ over $\mathcal{A}$. Note that if $\mathfrak{q}$ is a Boolean $\mathrm{CQ}$, then we have either $\operatorname{cert}(Q, \mathcal{A})=\{()\}$ if $(\mathcal{T}, \Sigma, \mathcal{A}) \models \mathfrak{q}()$, or $\operatorname{cert}(Q, \mathcal{A})=\{\}$ otherwise.

The decision problem associated to answering OMQs is the following: given an OMQ $Q$, a (possibly empty) tuple of individuals $\vec{a}$, and an $\mathrm{ABox} \mathcal{A}$, decide whether $\vec{a} \in \operatorname{cert}(Q, \mathcal{A})$. All complexity bounds in this paper are for this decision problem.

1. The normal form in [50] allows for axioms $\exists r . A \sqsubseteq B$, which can be rewritten as $A \sqsubseteq \forall r^{-} . B$. 
We remark that individuals are not allowed in queries, but this is not a limitation. To simulate them in an OMQ $Q=(\mathcal{T}, \Sigma, \mathfrak{q})$ we can use a fresh $A_{a} \in \mathrm{N}_{\mathrm{C}}$ and a fresh $x_{a} \in \mathrm{N}_{\mathrm{V}}$ for each $a \in \mathrm{N}_{\mathrm{I}}$ that occurs in $\mathfrak{q}$. If the atom $A_{a}\left(x_{a}\right)$ is added to $\mathfrak{q}$, and the axiom $\{a\} \sqsubseteq A_{a}$ is added to $\mathcal{T}$, then each occurrence of $a$ can be replaced by $x_{a}$.

In the OMQ literature, CQs are very prominent, and most research so far has focused on such OMQs and their extensions. It is well known that answering such OMQs is a 2EXPTIME complete problem whenever the TBox $\mathcal{T}$ is in any fragment of $\mathcal{A L C H O I}$ containing $\mathcal{A L C I}$ [37] or $\mathcal{A L C O}$ [42]. In the presence of closed predicates, 2EXPTIME-hardness holds already for TBoxes in the so-called $\mathcal{E} \mathcal{L}$ and DL-Lite logics, both of which are very restricted fragments of $\mathcal{A L C H \mathcal { H }}$.

Theorem 1. [37, 42] Answering an $O M Q Q=(\mathcal{T}, \Sigma, \mathfrak{q})$, where $\mathfrak{q}$ is a $C Q$ is 2EXPTIME-hard in the following cases:

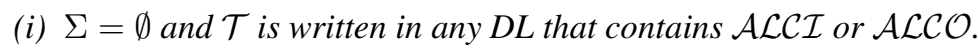

(ii) $\Sigma \neq \emptyset$ and $\mathcal{T}$ is written in any DL that contains $\mathcal{E} \mathcal{L}$ or DL-Lite $\mathcal{R}_{\text {. }}$

In this paper we want to show rewritability in polynomial time into DATALOG variants for classes of OMQs. But even the richest DATALOG variants we consider allow to decide entailment in coNEXPTIME ${ }^{\text {NP }}$, so the existence of a polynomial rewriting for the classes of OMQs mentioned in Theorem 1 would imply 2 EXPTIME $\subseteq$ coNEXPTIME $^{\mathrm{NP}}$, contradicting usual assumptions in complexity theory. This rules out these classes as potential candidates for polynomial rewritings; note that, in particular, by item (ii), we cannot have CQs for any non-trivial DL if we want to have closed predicates. For this reason, we need to consider more restricted classes of OMQs. We consider below a relaxation of the class of acyclic CQs, but first we define a class of queries - all whose variables are mapped to individuals — that we call c-safe, and that strictly generalizes instance queries.

In what follows, we may write $r \in \mathcal{T} \Sigma$ as a shorthand for $r \sqsubseteq \mathcal{T}^{*} s$ for some $s^{(-)} \in \Sigma$, that is, $r$ is subsumed by some closed predicate in $\mathcal{T}$.

Definition 4 (c-variables, c-safe CQs). Consider an OMQ $Q=(\mathcal{T}, \Sigma, \mathfrak{q})$ where $\mathfrak{q}$ is a CQ. We call $x$ a $c$-variable (in $Q$ ) if it is an answer variable of $\mathfrak{q}$, or if at least one of the following holds:

- there exists some atom $r(x, y)$ or $r(y, x)$ in $\mathfrak{q}$ such that $r \in \mathcal{T} \Sigma$, or

- there exists some atom $A(x)$ in $\mathfrak{q}$ such that $A \in \Sigma$.

We call $Q$ a $c$-safe query if all its variables are c-variables.

The two conditions in the definition above ensure that to answer a c-safe OMQ we only need to consider mappings of the variables to the individuals that occur explicitly in the input ABox. We also consider a more general class of queries that allows for conjunctions and existentially quantified variables provided they only participate in cycles in a restricted way. It is a slight generalization of the usual acyclicity condition for CQs [52].

Definition 5 (c-acyclic CQs). Consider an OMQ $Q=(\mathcal{T}, \Omega, \mathfrak{q})$, where $\mathfrak{q}$ is a CQ such that each $\alpha \in \mathfrak{q}$ is an atom with terms from $N_{\vee}$. The query graph (a.k.a. the Gaifman graph) $G(\mathfrak{q})$ of $\mathfrak{q}$ is the undirected graph whose nodes are the variables of $\mathfrak{q}$, and that has an edge between $x$ and $y$ if they occur together in some atom in $\mathfrak{q}$. The connected components of $\mathfrak{q}$ are those of $G(\mathfrak{q})$, and $\mathfrak{q}$ is connected if $G(\mathfrak{q})$ is. We call a CQ $\mathfrak{q}$ acyclic if $G(\mathfrak{q})$ is acyclic, and for every edge $(x, y)$ in $G(\mathfrak{q})$, there is exactly one atom in $\mathfrak{q}$ of the form $r(x, y)$ or $r(y, x)$.

We call $Q=(\mathcal{T}, \Omega, \mathfrak{q})$ acyclic modulo $c$-variables (c-acyclic for short) if the query $\mathfrak{q}^{-}$obtained by dropping all atoms $r(x, y)$ where both $x$ and $y$ are $c$-variables is acyclic, and every connected component in $G\left(\mathfrak{q}^{-}\right)$has one c-variable.

To answer c-acyclic OMQs we reduce them to c-safe OMQs with the same answers, using the so-called rolling up technique, which essentially replaces by a complex concept each acyclic query component that contains one c-variable. 
Definition 6. Let $\mathfrak{q}$ be a connected, acyclic CQ, and let $x_{0} \in \operatorname{vars}(\mathfrak{q})$. We denote by $T_{q, x_{0}}$ the tree that results from $G(\mathfrak{q})$ by taking $x_{0}$ as a root. We inductively assign to each variable $x \in \operatorname{vars}(\mathfrak{q})$ a concept $C_{\mathfrak{q}, x}$ as follows:

- if $x$ is a leaf node then $C_{\mathfrak{q}, x}=\prod_{C(x) \in \mathfrak{q}} C$,

- if the children of the node $x$ are $x_{1}, \ldots, x_{n}$, then

$$
C_{\mathfrak{q}, x}=\prod_{C(x) \in \mathfrak{q}} C \sqcap \prod_{\substack{1 \leq i \leq n \\ r\left(x, x_{i}\right) \in \mathfrak{q} \text { or } r^{-}\left(x_{i}, x\right) \in \mathfrak{q}}} \exists r \cdot C_{\mathfrak{q}, x_{i}}
$$

We call $C_{\mathfrak{q}, x_{0}}$ the query concept of $\mathfrak{q}$ w.r.t. $x_{0}$.

The concept $C_{\mathfrak{q}, x}$ is in a fragment of $\mathcal{A L C H O I}$ called $\mathcal{E} \mathcal{L} \mathcal{I}$, and it correctly captures the semantics of the CQ q.

Lemma 1. [31] Let $\mathfrak{q}$ be a connected, acyclic CQ. Let $x \in \operatorname{vars}(\mathfrak{q})$, and let $C_{\mathfrak{q}, x}$ be the query concept of $\mathfrak{q}$ w.r.t. $x$. Then for every interpretation $\mathcal{I}=\left\langle\Delta^{\mathcal{I}},{ }^{\mathcal{I}}\right\rangle$ and any object $d \in \Delta^{\mathcal{I}}$, the following are equivalent:

- $d \in C_{\mathfrak{q}, x}^{\mathcal{I}}$,

- there is some $\pi$ such that $\mathcal{I} \models \pi(\mathfrak{q})$ with $\pi(x)=d$.

Every c-acyclic OMQ $Q$ can be transformed into a c-safe OMQ $Q^{\prime}$ that contains the c-variables of $Q$ and that has the same certain answers as $Q$.

Lemma 2. Consider a c-acyclic $O M Q Q=(\mathcal{T}, \Sigma, \mathfrak{q})$ and let $X$ be the set of c-variables of $Q$. Then $Q$ can be transformed in polynomial time into an $O M Q Q^{\prime}=\left(\mathcal{T}^{\prime}, \Sigma, \mathfrak{q}^{\prime}\right)$, such that $\mathfrak{q}^{\prime}$ is a CQ with vars $\left(\mathfrak{q}^{\prime}\right)=X$ and for every ABox $\mathcal{A}$ over the concept and role names occurring in $Q$ the following holds:

$$
\operatorname{cert}(Q, \mathcal{A})=\operatorname{cert}\left(Q^{\prime}, \mathcal{A}\right)
$$

Proof (Sketch). The transformation of $Q$ to $Q^{\prime}$ is as follows. We associate to $\mathfrak{q}$ the set of connected components $\left\{T_{1}, \ldots, T_{n}\right\}$ of $G^{-}(\mathfrak{q})$. Because $Q$ is c-acyclic, every $T_{i}$ is a connected acyclic undirected graph containing exactly one node $x_{i}$ that is a c-variable. We designate $x_{i}$ as the root of $T_{i}$, allowing us to view $T_{i}$ as a tree and we denote by $C_{T_{i}, x_{i}}$ the query concept of $T_{i}$ w.r.t. $x_{i}$.

The new OMQ $Q^{\prime}=\left(\mathcal{T}^{\prime}, \Sigma, \mathfrak{q}^{\prime}(\vec{x})\right)$ is defined as follows:

$$
\begin{aligned}
\mathcal{T}^{\prime} & =\mathcal{T} \cup\left\{C_{T_{i}, x_{i}} \sqsubseteq A_{T_{i}} \mid T_{i} \in\left\{T_{1}, \ldots, T_{n}\right\}\right\} \\
\mathfrak{q}^{\prime}(\vec{x}) & =\bigwedge_{T_{i} \in\left\{T_{1}, \ldots, T_{n}\right\}} A_{T_{i}}\left(x_{i}\right) \wedge \bigwedge_{\left\{x_{i}, x_{j}\right\} \subseteq X, r\left(x_{i}, x_{j}\right) \in \mathfrak{q}} r\left(x_{i}, x_{j}\right)
\end{aligned}
$$

where each $A_{T_{i}}$ is a fresh concept name. Using Lemma 1 it can be easily verified that for any ABox $\mathcal{A}$ over the concept and role names that appear in the OMQ $Q$, the queries $Q$ and $Q^{\prime}$ produce the same certain answers.

\section{Game Characterization of Countermodels}

Consider a c-safe OMQ $Q=(\mathcal{T}, \Sigma, \mathfrak{q}(\vec{x}))$, where $\mathcal{T}$ is in normal form, $\mathcal{A}$ is an ABox over the concept and role names occurring in $\mathcal{T}$, and let $\mathcal{K}=(\mathcal{T}, \Sigma, \mathcal{A})$. To decide $\mathcal{K} \forall \mathfrak{q}(\vec{a})$ for some tuple of individuals $\vec{a}$ occurring in $\mathcal{K}$, we will use the notion of a core, which is essentially an interpretation whose domain is $\mathrm{N}_{\mathrm{I}}(\mathcal{K})$ extended with a small number of additional individuals. Cores fix how the individuals of $\mathcal{K}$ participate in concepts and roles, ensuring the non-entailment of $\mathfrak{q}(\vec{a})$, the satisfaction of $\mathcal{A}$ and $\Sigma$, and a partial satisfaction of $\mathcal{T}$. To decide whether there exists an interpretation $\mathcal{I}$ such that $\mathcal{I} \models \mathcal{K}$ and $\mathcal{I} \not \models \mathfrak{q}(\vec{a})$, we will proceed in two steps: 
(1) Guess a core $\mathcal{I}_{c}$ for $\mathcal{K}$ such that $\mathcal{I}_{c} \forall \models \mathfrak{q}(\vec{a})$.

(2) Check that $\mathcal{I}_{c}$ can be extended to satisfy all axioms in $\mathcal{T}$.

We start by formally defining cores.

Definition 7. For each individual $c$, and an existential inclusion $\alpha$ of type (N2), we assume a special constant $c^{\alpha}$ that is prohibited to occur in ABoxes. Such individuals are called fringe individuals. Assume a KB $\mathcal{K}=(\mathcal{T}, \Sigma, \mathcal{A})$. We let $c \operatorname{dom}(\mathcal{K})$ contain the individuals $c, c^{\alpha}$ for all $c \in \mathrm{N}_{\mathrm{I}}(\mathcal{K})$, and existential inclusions $\alpha$ in $\mathcal{T}$. A core for $\mathcal{K}$ is an interpretation $\mathcal{I}_{c}=\left(\Delta^{\mathcal{I}_{c}},{ }^{\mathcal{I}_{c}}\right)$ such that

(c1) $\Delta^{\mathcal{I}_{c}} \subseteq \operatorname{cdom}(\mathcal{K}), \mathrm{N}_{\mathrm{I}}(\mathcal{K}) \subseteq \Delta^{\mathcal{I}_{c}}$, and $a^{\mathcal{I}_{c}}=a$ for all $a \in \mathrm{N}_{\mathrm{I}}(\mathcal{K})$,

(c2) $\mathcal{I}_{c} \models_{\Sigma} \mathcal{A}$,

(c3) $\mathcal{I}_{c} \models \alpha$ for each $\alpha \in \mathcal{T}$ such that

(c3.1) $\alpha$ is of the form $B_{1} \sqcap \cdots \sqcap B_{n} \sqsubseteq B_{n+1} \sqcup \cdots \sqcup B_{k} \in \mathcal{T}$,

(c3.2) $\alpha$ is of the form $A \sqsubseteq \forall r . A^{\prime}$,

(c3.3) $\alpha$ is of the form $r \sqsubseteq s$,

(c3.4) $\alpha$ is of the form $A \sqsubseteq \exists r . A^{\prime}$ and $r \in_{\mathcal{T}} \Sigma$, or

(c4) if $\left(d, d^{\prime}\right) \in r^{\mathcal{I}_{c}}$, then one of the following holds:

- $\left\{d, d^{\prime}\right\} \subseteq \mathrm{N}_{\mathrm{I}}(\mathcal{K})$,

- $d \in \mathrm{N}_{\mathrm{I}}(\mathcal{K})$ and $d^{\prime}=d^{\alpha}$ for some $\alpha \in \mathcal{T}$, or

- $d^{\prime} \in \mathrm{N}_{\mathrm{I}}(\mathcal{K})$ and $d=d^{\prime \alpha}$ for some $\alpha \in \mathcal{T}$.

(c5) if $d \in \Delta^{\mathcal{I}_{c}}$ is not a fringe individual, then $d \in A^{\mathcal{I}_{c}}$ implies $d \in\left(\exists r . A^{\prime}\right)^{\mathcal{I}_{c}}$ for every $A \sqsubseteq \exists r . A^{\prime} \in \mathcal{T}$.

The conditions in the above definition can be explained as follows. The domain of a core interpretation $\mathcal{I}_{c}$ for a $\mathrm{KB} \mathcal{K}$ always contains the individuals in $\mathcal{K}$, and possibly some special fringe individuals (condition (c1)). The condition (c2) tells us that $\mathcal{I}_{c}$ must satisfy the ABox assertions in $\mathcal{K}$ and respect the closed predicates. The condition (c3) requires all TBox inclusions to be satisfied, with the possible exception of existential axioms $A \sqsubseteq \exists r$. $A^{\prime}$ such that $r \notin_{\mathcal{T}} \Sigma$. Using the condition (c4) we restrict the possible connection between the domain elements. In particular, we allow a fringe individual $c^{\alpha}$ to be connected by a role only to its "parent" individual $c$. Intuitively, the condition (c5) makes sure that the original ABox individuals satisfy all TBox inclusions. Thus, intuitively, the only reason for $\mathcal{I}_{c}$ to not be a model of the TBox is a fringe individual that triggers an existential inclusion $A \sqsubseteq \exists r . A^{\prime}$ with $r \notin_{\mathcal{T}} \Sigma$. The goal of the game-like characterization is to see how a proper core interpretation can be extended to satisfy all existential inclusions. The notion of an extension is as expected:

Definition 8. Consider a core $\mathcal{I}_{c}$ for $\mathcal{K}=(\mathcal{T}, \Sigma, \mathcal{A})$. An interpretation $\mathcal{J}$ is called an extension of $\mathcal{I}_{c}$, if the following hold:

(i) $\Delta^{\mathcal{I}_{c}} \subseteq \Delta^{\mathcal{J}}$,

(ii) for all concept names $A, A^{\mathcal{I}_{c}}=A^{\mathcal{J}} \cap \Delta^{\mathcal{I}_{c}}$,

(iii) for all role names $p, p^{\mathcal{I}_{c}}=p^{\mathcal{J}} \cap\left(\Delta^{\mathcal{I}_{c}} \times \Delta^{\mathcal{I}_{c}}\right)$, and

(iv) $q^{\mathcal{J}}=q^{\mathcal{I}_{c}}$ for all $q \in \Sigma$. 

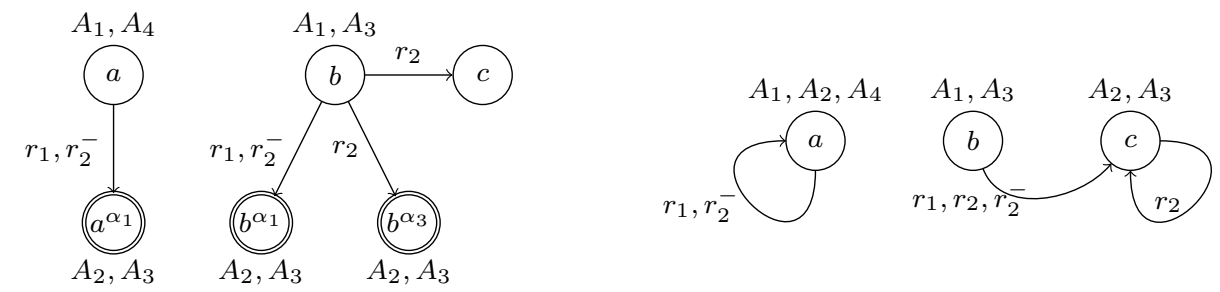

Figure 1: Example cores $\mathcal{I}_{c}^{1}$ and $\mathcal{I}_{c}^{2}$ for the $\mathrm{KB}$ in Example 1

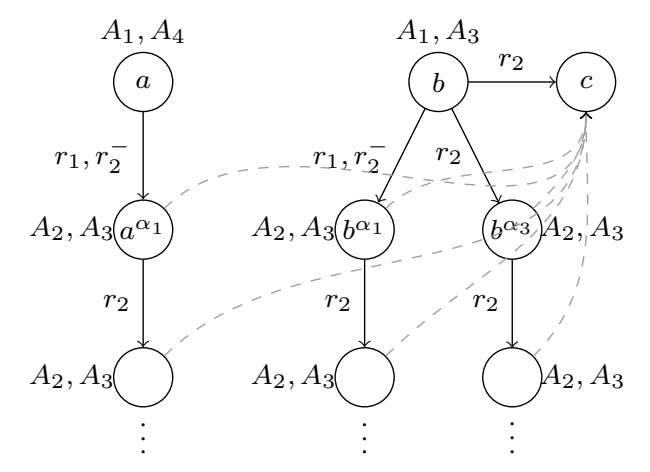

Figure 2: A model of $\mathcal{K}$ that extends the core $\mathcal{I}_{c}^{1}$.

Example 1. Consider the $\mathrm{KB} \mathcal{K}=(\mathcal{T}, \mathcal{A}, \Sigma)$, where $\Sigma=\left\{A_{1}, A_{4}\right\}$, the TBox $\mathcal{T}$ contains the following inclusions:

$$
\begin{array}{ll}
\alpha_{1}=A_{1} \sqsubseteq \exists r_{1} \cdot A_{2} & \alpha_{4}=A_{4} \sqsubseteq \forall r_{2} \cdot A_{1} \\
\alpha_{2}=A_{2} \sqsubseteq A_{3} \sqcup A_{4} & \alpha_{5}=A_{3} \sqsubseteq \exists r_{2} \cdot\{c\} \\
\alpha_{3}=A_{3} \sqsubseteq \exists r_{2} \cdot A_{2} & \alpha_{6}=r_{1}^{-} \sqsubseteq r_{2}
\end{array}
$$

and the $\mathrm{ABox} \mathcal{A}$ is as follows:

$$
\mathcal{A}=\left\{A_{1}(a), \quad A_{4}(a), \quad A_{1}(b), \quad A_{3}(b)\right\}
$$

The interpretations $\mathcal{I}_{c}^{1}$ and $\mathcal{I}_{c}^{2}$ in Figure 1 are both cores for $\mathcal{I}_{c}$. The fringe individuals are depicted with double circles.

The core $\mathcal{I}_{c}^{2}$ on the right is itself a model. The core $\mathcal{I}_{c}^{1}$ is not, since the fringe individuals do not satisfy $\alpha_{3}$ and $\alpha_{5}$, but we can extend it into a model. For example, we can take its infinite extension by adding to each fringe individual an $r_{2}$-child that satisfies $A_{2}$ and $A_{3}$, which is connected via $r_{2}$ to $c$, and repeating the same way for all the introduced successors, see Figure 2 The $r_{2}$-connections to $c$ are dashed grey and unlabeled for readability.

A core and its extensions coincide on the assertions they satisfy over the individuals occurring in the input $\mathrm{KB}$, and the non-entailment of a set of assertions from a KB is always witnessed by some core that can be extended into a model.

Lemma 3. Assume a c-safe $O M Q Q=(\mathcal{T}, \Sigma, \mathfrak{q}(\vec{x}))$ and a $K B \mathcal{K}=(\mathcal{T}, \Sigma, \mathcal{A})$. Then $\mathcal{K} \forall \mathfrak{q}(\vec{a})$ for some tuple of individuals $\vec{a}$ occurring in $\mathcal{K}$ iff there exists a core $\mathcal{I}_{c}$ for $\mathcal{K}$ such that 
(1) $\mathcal{I}_{c} \not \models \mathfrak{q}(\vec{a})$, and

(2) there exists an extension $\mathcal{J}$ of $\mathcal{I}_{c}$ such that $\mathcal{J} \models \mathcal{K}$.

Proof. The " $\Leftarrow$ " direction is not difficult. Observe that if $\mathcal{J}$ is an extension of $\mathcal{I}_{c}$, then $\mathcal{J}$ and $\mathcal{I}_{c}$ agree on the participation of constants from $\mathcal{K}$ in all concept and role names. I.e., for all constants $c, d \in \mathrm{N}_{\mathrm{I}}(\mathcal{K})$ and all concept names $A$ and all role names $r$, we have $c \in A^{\mathcal{J}}$ iff $c \in A^{\mathcal{I}_{c}}$, and $(c, d) \in r^{\mathcal{J}}$ iff $(c, d) \in r^{\mathcal{I}_{c}}$. This observation, together with the facts that $Q$ is c-safe and $\mathcal{J} \models \mathcal{K}$, implies that $\mathcal{J} \not \models q(\vec{a})$, and, hence, $\mathcal{K} \not \models \mathfrak{q}(\vec{a})$.

For the " $\Rightarrow$ " direction, let $\mathcal{I}$ be an interpretation such that $\mathcal{I} \models \mathcal{K}$ and $\mathcal{I} \not \models \mathfrak{q}(\vec{a})$. W.l.o.g. we assume $q^{\mathcal{I}}=\emptyset$ for all $q \notin \mathrm{N}_{\mathrm{C}}(\mathcal{K}) \cup \mathrm{N}_{\mathrm{R}}(\mathcal{K})$. For every element $e \in \Delta^{\mathcal{I}}$ and every inclusion $\alpha=A \sqsubseteq \exists r . A^{\prime} \in \mathcal{T}$ with $e \in A^{\mathcal{I}}$, let wit $(e, \alpha)$ denote an arbitrary but fixed element $e^{\prime} \in \Delta^{\mathcal{I}}$ with $\left(e, e^{\prime}\right) \in r^{\mathcal{I}}$ and $e^{\prime} \in\left(A^{\prime}\right)^{\mathcal{I}}$. Note that $e^{\prime}$ always exists because $\mathcal{I}$ is a model of $\mathcal{K}$. We build now the "unravelling" of $\mathcal{I}$. For a word $w$ of form $c \cdot\left(\alpha_{1}, e_{1}\right) \cdots\left(\alpha_{n}, e_{n}\right)$, we let $\operatorname{tail}(w)=c$ in case $n=0$, and $\operatorname{tail}(w)=e_{n}$ in case $n>0$. Let Paths be the $\subseteq$-minimal set of words that includes $\mathrm{N}_{\mathrm{I}}(\mathcal{K})$, and satisfies the following rule: if $w \in$ Paths, $\alpha$ is an existential inclusion in $\mathcal{T}, e=\operatorname{wit}(\operatorname{tail}(w), \alpha)$ is defined, and $e \notin \mathrm{N}_{\mathrm{l}}(\mathcal{K})$, then $w \cdot(\alpha, e) \in$ Paths. We unravel $\mathcal{I}$ into an interpretation $\mathcal{U}$. More precisely, $\mathcal{U}$ is defined as follows:

- $\Delta^{\mathcal{U}}=$ Paths, and $c^{\mathcal{U}}=c$ for all $c \in \mathrm{N}_{\mathrm{I}}(\mathcal{K})$,

- for all concept names $A, A^{\mathcal{U}}=\left\{w \in \Delta^{\mathcal{U}} \mid \operatorname{tail}(w) \in A^{\mathcal{I}}\right\}$,

- for all role names $r, r^{\mathcal{U}}=\left\{\left(w, w^{\prime}\right) \in \Delta^{\mathcal{U}} \times \Delta^{\mathcal{U}} \mid\left(\operatorname{tail}(w), \operatorname{tail}\left(w^{\prime}\right)\right) \in r^{\mathcal{I}}\right\}$.

It is standard to see that $\mathcal{U} \models \mathcal{K}$ and $\mathcal{U} \forall \mathfrak{q}(\vec{a})$. A core $\mathcal{I}_{c}$ can be obtained from $\mathcal{U}$ in two steps. First, we restrict the domain of $\mathcal{U}$ to paths of length at most 1, i.e. to domain elements of the form $c$ and $c \cdot\left(\alpha_{1}, v_{1}\right)$. Second, all domain elements $c \cdot\left(\alpha_{1}, v_{1}\right)$ are replaced by the constant $c^{\alpha_{1}}$. Note that since $w \cdot\left(\alpha, v_{1}\right) \in$ Paths and $w \cdot\left(\alpha, v_{2}\right) \in$ Paths implies $v_{1}=v_{2}$, the second step is simply a renaming of domain elements, i.e. the second step produces an isomorphic interpretation. The satisfaction of the conditions (c1-c6) by $\mathcal{I}_{c}$ is ensured because $\mathcal{U}$ is a model of $\mathcal{K}$. Since $\mathcal{I}_{c}$ is obtained by restricting $\mathcal{U}$ and $\mathcal{U} \forall \forall \mathfrak{q}(\vec{a})$, we have $\mathcal{I}_{c} \not \models \mathfrak{q}(\vec{a})$ (condition (11). Furthermore, $\mathcal{U}$ is the desired extension of $\mathcal{I}_{c}$ that is a model of $\mathcal{K}$ (condition (2)).

By this lemma, deciding non-entailment of a c-safe query amounts to deciding whether there is a core that does not satisfy it, and that can be extended into a model. Since the domain of cores is bounded, it is not hard to achieve this with a polynomially sized program. However, verifying whether a core can be extended into a full model is hard, as it corresponds to testing consistency (of $\mathcal{I}_{c}$ viewed as an ABox) with respect to $\mathcal{T}$, an EXPTIME-hard problem already for fragments of $\mathcal{A L C H O I}$ such as $\mathcal{A L C}$ [48]. In order to obtain a polynomial set of rules that solves this EXPTIME-hard problem, we first characterize it as a game, revealing a simple algorithm for it that admits an elegant implementation in non-monotonic disjunctive DATALOG. For this we use types, which we define as follows:

Definition 9. A type $\tau$ (over a TBox $\mathcal{T}$ ) is a subset of $\mathrm{N}_{\mathrm{C}}^{+}(\mathcal{T})$ such that $\perp \notin \tau$ and $T \in \tau$. We denote by $\operatorname{types}(\mathcal{T})$ the set of all types over $\mathcal{T}$.

We say that $\tau$ satisfies an inclusion $\alpha=B_{1} \sqcap \cdots \sqcap B_{n} \sqsubseteq B_{n+1} \sqcup \cdots \sqcup B_{k}$ of type (N1), if $\left\{B_{1}, \ldots, B_{n}\right\} \subseteq$ $\tau$ implies $\left\{B_{n+1}, \ldots, B_{k}\right\} \cap \tau \neq \emptyset$; otherwise $\tau$ violates $\alpha$. Let $\mathcal{I}$ be an interpretation. For an element $e \in \Delta^{\mathcal{I}}$, we let type $(e, \mathcal{I})=\left\{B \in \mathrm{N}_{\mathrm{C}}^{+}(\mathcal{T}) \mid e \in B^{\mathcal{I}}\right\}$. A type $\tau$ is realized by e (in $\left.\mathcal{I}\right)$ if type $(e, \mathcal{I})=\tau$; we say that $\tau$ is realized in $\mathcal{I}$ if it is realized by some $e \in \Delta^{\mathcal{I}}$.

\section{The Model Building Game}

We now describe a simple game to decide whether a given core $\mathcal{I}_{c}$ can be extended into a model of a KB $\mathcal{K}$. The game is played by Bob (the builder), who wants to extend $\mathcal{I}_{c}$ into a model, and Sam (the spoiler), who wants to spoil all Bob's attempts. Intuitively speaking, Sam starts the game by picking a fringe individual $a$ in $\mathcal{I}_{c}$, whose type type $\left(a, \mathcal{I}_{c}\right)$ becomes the current type. Next, Sam chooses an inclusion of the form 
$A \sqsubseteq \exists r . A^{\prime}$ that is "triggered" by the current type. It is then the turn of Bob to respond by selecting a type for the corresponding $r$-successor that satisfies $A^{\prime}$. The game continues for as long as Bob can respond to the challenges of Sam, where at each further round the response by Bob is set to be the current type in the game.

We use the term $c$-type to refer to a type $\tau \in \operatorname{types}(\mathcal{T})$ such that $\tau$ contains a nominal $\{a\}, \tau \cap \Sigma \neq \emptyset$, or $A \in \tau$ for some $A \sqsubseteq \exists r . A^{\prime} \in \mathcal{T}$ with $r \in \mathcal{T} \quad \Sigma$. Note that, in any model, these types can only be realized by the interpretation of individuals in $\mathrm{N}_{1}$.

For a TBox $\mathcal{T}$, a set $\Sigma \subseteq \mathrm{N}_{\mathrm{C}} \cup \mathrm{N}_{\mathrm{R}}$ and a core $\mathcal{I}_{c}$ for $\mathcal{K}=(\mathcal{T}, \Sigma, \mathcal{A})$, where $\mathcal{A}$ is an ABox over the concept and role names occurring in $\mathcal{T}$, we define the locally consistent set $\operatorname{LC}\left(\mathcal{T}, \Sigma, \mathcal{I}_{c}\right)$ as the set of types $\tau \in \operatorname{types}(\mathcal{T})$ such that:

$\left(L C_{\mathrm{N} 1}\right) \tau$ satisfies all inclusions of type $(\mathrm{N} 1)$ in $\mathcal{T}$.

$\left(L C_{\Sigma}\right)$ If $\tau$ is a c-type, then $\tau$ must be realized in $\mathcal{I}_{c}$.

The game is played using types from $\operatorname{LC}\left(\mathcal{T}, \Sigma, \mathcal{I}_{c}\right)$ only. Note that, since $\mathcal{I}_{c}$ is a core, a c-type can only be realized by an individual in $N_{I}(\mathcal{K})$ and, by definition, the individuals in a core already satisfy all axioms. We are now ready to describe the game.

The model building game Consider a core $\mathcal{I}_{c}$ for $\mathcal{K}$. The game on $\mathcal{I}_{c}$ starts by Sam choosing a fringe individual $a \in \Delta^{\mathcal{I}_{c}}$, and $\tau=\operatorname{type}\left(a, \mathcal{I}_{c}\right)$ is set to be the current type. Then:

(§) Sam chooses an inclusion $A \sqsubseteq \exists r . A^{\prime} \in \mathcal{T}$ such that $A \in \tau$. If there is no such inclusion, the game is over and Bob wins. Otherwise, Bob chooses a type $\tau^{\prime} \in \operatorname{LC}\left(\mathcal{T}, \Sigma, \mathcal{I}_{c}\right)$ such that:

(C1) $A^{\prime} \in \tau^{\prime}$, and

(C2) for all inclusions $A_{1} \sqsubseteq \forall s . A_{2} \in \mathcal{T}$ :

$$
\begin{aligned}
& \text { - if } r \sqsubseteq_{\mathcal{T}}^{*} s \text { and } A_{1} \in \tau \text { then } A_{2} \in \tau^{\prime}, \\
& \text { - if } r^{-} \sqsubseteq_{\mathcal{T}}^{*} s \text { and } A_{1} \in \tau^{\prime} \text { then } A_{2} \in \tau .
\end{aligned}
$$

If $\tau^{\prime}$ does not exist, then Sam wins the game. If $\tau^{\prime}$ exists and is a c-type, then Bob wins the game. If $\tau^{\prime}$ exists but is not a c-type, then $\tau^{\prime}$ is set to be the current type and the game continues with a new round, i.e. we go back to $\diamond$.

We now illustrate the game. To avoid clutter, we omit $\top$ from all types.

Example 2. Sam and Bob play on $\mathcal{I}_{c}^{1}$ as follows. Assume that Sam starts by picking the fringe individual $a^{\alpha_{1}}$, hence $\tau=\left\{A_{2}, A_{3}\right\}$ is the current type. The cases of $b^{\alpha_{1}}$ and $b^{\alpha_{3}}$ are the same.

From $\tau=\left\{A_{2}, A_{3}\right\}$, Sam can choose either $\alpha_{3}$ or $\alpha_{5}$ (see Figure 3). In the latter case, Bob can win the game by picking the type $\{\{c\}\}$. In the former case, Bob can pick $\tau=\left\{A_{2}, A_{3}\right\}$, which contains $A_{2}$ and satisfies $\mathrm{LC}\left(\mathcal{T}, \Sigma, \mathcal{I}_{c}^{1}\right)$. Note that $\tau$ is Bob's only good choice: by $\alpha_{2}$, any type in $\operatorname{LC}\left(\mathcal{T}, \Sigma, \mathcal{I}_{c}^{1}\right)$ that contains $A_{2}$ must contain $A_{3}$ or $A_{4}$, but $A_{4} \in \Sigma$, and there is no type containing both $A_{2}$ and $A_{4}$ realized in $\mathcal{I}_{c}^{1}$. So $\tau$ is the only type in $\operatorname{LC}\left(\mathcal{T}, \Sigma, \mathcal{I}_{c}^{1}\right)$ containing $A_{2}$.

Since $\tau=\left\{A_{2}, A_{3}\right\}$ is not a c-type, the game continues from this type, and we are back to the same situation. Sam can pick $\alpha_{3}$ or $\alpha_{5}$. If he picks $\alpha_{5}$ Bob can win by picking $\{\{c\}\}$. If he picks $\alpha_{3}$, Bob can again respond with $\tau=\left\{A_{2}, A_{3}\right\}$. The game continues for as long as Sam keeps choosing $\alpha_{3}$, and Bob never loses.

\section{Runs and strategies}

We define runs and strategies. Intuitively, a run starts at a fringe individual $a$ picked initially by Sam, and then it comprises a (possibly infinite) sequence of inclusions $\alpha_{i}$ picked by Sam in round $i$, and types $\tau_{i}$ picked by Bob in response. A strategy gives a move for Bob in response to each possible previous move of Sam, and it is called non-losing if it guarantees that Sam does not defeat him. 


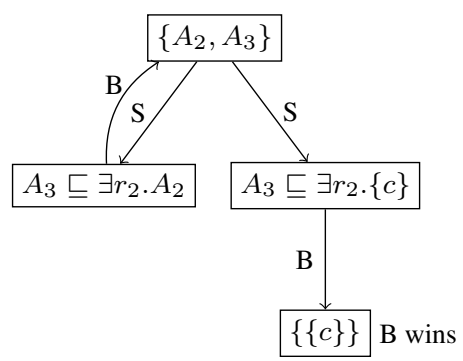

Figure 3: Non-losing strategy for Bob on the core $\mathcal{I}_{c}^{1}$. (S stands for Sam, B for Bob)

Definition 10. A run of the game on a core $\mathcal{I}_{c}$ for $\mathcal{K}$ is a (possibly infinite) sequence

$$
a \alpha_{1} \tau_{1} \alpha_{2} \tau_{2} \ldots
$$

where $i \geq 0, a$ is a fringe individual in $\mathcal{I}_{c}$, each $\alpha_{i}$ is an existential inclusion in $\mathcal{T}$, each $\tau_{i}$ is a type over $\mathcal{T}$, and either there are no c-types in it, or $a \alpha_{1} \tau_{1} \alpha_{2} \tau_{2} \ldots \alpha_{\ell} \tau_{\ell}$ is finite and $\tau_{\ell}$ is the only c-type.

A strategy for Bob is a partial function str that maps each pair of a type $\tau$ that is not a c-type and an inclusion $A \sqsubseteq \exists r . A^{\prime} \in \mathcal{T}$ with $A \in \tau$, to a type $\tau^{\prime}$ that satisfies (C1) and (C2). A run $a \alpha_{1} \tau_{1} \alpha_{2} \tau_{2} \ldots$ follows a strategy str, if $\tau_{0}=\operatorname{type}\left(a, \mathcal{I}_{c}\right)$, and $\tau_{i}=\operatorname{str}\left(\tau_{i-1}, \alpha_{i}\right)$ for every $i>0$.

For a finite run $w$, we let $\operatorname{tail}(w)=\operatorname{type}\left(a, \mathcal{I}_{c}\right)$ if $w=a$, and $\operatorname{tail}(w)=\tau_{\ell}$ if $w=a \ldots \alpha_{\ell} \tau_{\ell}$ with $\ell \geq 1$. The strategy str is called non-losing on $\mathcal{I}_{c}$ if for every finite run $w$ that follows $\operatorname{str}, \operatorname{tail}(w) \in \operatorname{LC}\left(\mathcal{T}, \Sigma, \mathcal{I}_{c}\right)$ and $\operatorname{str}\left(\operatorname{tail}(w), A \sqsubseteq \exists r . A^{\prime}\right)$ is defined for every $\operatorname{tail}(w)$ that is not a c-type and every $A \sqsubseteq \exists r . A^{\prime} \in \mathcal{T}$ such that $A \in \operatorname{tail}(w)$.

Note that there are no runs on a core that has no fringe individuals, and therefore, every strategy is nonlosing on it.

Example 3. Figure 3 depicts the following non-losing strategy str for Bob $\mathcal{I}_{c}^{1}$ :

$$
\begin{aligned}
\operatorname{str}\left(\left\{A_{2}, A_{3}\right\}, A_{3} \sqsubseteq \exists r_{2} . A_{2}\right) & =\left\{A_{1}, A_{2}, A_{4}\right\} \\
\operatorname{str}\left(\left\{A_{2}, A_{3}\right\}, A_{3} \sqsubseteq \exists r_{2} .\{c\}\right) & =\{\{c\}\} .
\end{aligned}
$$

Note that for $\mathcal{I}_{c}^{2}$ every strategy is winning since there are no fringe individuals.

\section{Correctness of the game characterization}

The following proposition establishes that the existence of a non-losing strategy for Bob on a given core correctly characterizes the existence of a model of $\mathcal{K}$ extending that core.

Proposition 2. Consider a $K B \mathcal{K}$ and a core $\mathcal{I}_{c}$ for $\mathcal{K}$. There is a non-losing strategy str for Bob on $\mathcal{I}_{c}$ iff $\mathcal{I}_{c}$ can be extended into a model $\mathcal{J}$ of $\mathcal{K}$.

Proof. For the " $\Rightarrow$ " direction, from an arbitrary non-losing str for Bob on $\mathcal{I}_{c}$, we build an interpretation $\mathcal{J}$ as follows.

For each c-type $\tau$ that is realized in $\mathcal{I}_{c}$, we let $a_{\tau}$ denote a fixed, arbitrary individual realizing $\tau$; in particular, $a_{\tau}=b$ if $\{b\} \in \tau$.

Let $S$ be the set of all finite runs that follow str on $\mathcal{I}_{c}$. We consider two subsets frn and $c r n$ of $S$ defined as follows:

$$
\begin{aligned}
\operatorname{frn}\left(\mathcal{I}_{c}, s t r\right)=\left\{a \alpha_{1} \tau_{1} \cdots \alpha_{\ell} \tau_{\ell} \in S \mid\right. & \text { each } \left.\tau_{i} \text { for } 1 \leq i \leq \ell \text { is not a c-type }\right\} \\
\operatorname{crn}\left(\mathcal{I}_{c}, s t r\right)=\left\{a \alpha_{1} \tau_{1} \cdots \alpha_{\ell} \tau_{\ell} \in S \mid\right. & \tau_{\ell} \text { is a c-type, and for each } 1 \leq i<\ell, \\
& \left.\tau_{i} \text { is not a c-type }\right\} .
\end{aligned}
$$


Roughly, frn collects all the finite runs from $S$ that do not contain any c-types and $c r n$ collects all those that end in a c-type.

We define the domain of the desired $\mathcal{J}$ :

$$
\Delta^{\mathcal{J}}=\mathrm{N}_{\mathrm{l}}(\mathcal{K}) \cup \operatorname{frn}\left(\mathcal{I}_{c}, s t r\right)
$$

As ${ }^{\mathcal{J}}$ we take an interpretation function that satisfies the following equalities, for all $a \in \mathrm{N}_{\mathrm{I}}(\mathcal{K})$, all $A \in \mathrm{N}_{\mathrm{C}}$, and all $p \in \mathrm{N}_{\mathrm{R}}$ :

$$
\begin{aligned}
a^{\mathcal{J}}=a & \\
A^{\mathcal{J}}=A^{\mathcal{I}_{c}} \cup & \left\{w \in \Delta^{\mathcal{J}} \mid A \in \operatorname{tail}(w)\right\} \\
p^{\mathcal{J}}=p^{\mathcal{I}_{c}} \cup & \left\{(w, w \alpha \tau) \in \Delta^{\mathcal{J}} \times \Delta^{\mathcal{J}} \mid r \sqsubseteq_{\mathcal{T}}^{*} p, \alpha=A \sqsubseteq \exists r . A^{\prime} \in \mathcal{T}\right\} \cup \\
& \left\{(w \alpha \tau, w) \in \Delta^{\mathcal{J}} \times \Delta^{\mathcal{J}} \mid r^{-} \sqsubseteq_{\mathcal{T}}^{*} p, \alpha=A \sqsubseteq \exists r . A^{\prime} \in \mathcal{T}\right\} \cup \\
& \left\{\left(w, a_{\tau}\right) \in \Delta^{\mathcal{J}} \times \Delta^{\mathcal{J}} \mid w \alpha \tau \in \operatorname{crn}\left(\mathcal{I}_{c}, s t r\right), r \sqsubseteq_{\mathcal{T}}^{*} p\right\} \cup \\
& \left\{\left(a_{\tau}, w\right) \in \Delta^{\mathcal{J}} \times \Delta^{\mathcal{J}} \mid w \alpha \tau \in \operatorname{crn}\left(\mathcal{I}_{c}, s t r\right), r^{-} \sqsubseteq_{\mathcal{T}}^{*} p\right\}
\end{aligned}
$$

Note that taking such an interpretation is possible, since $w \alpha \tau \in \operatorname{crn}\left(\mathcal{I}_{c}, s t r\right)$ implies that $\tau$ is a c-type in $\operatorname{LC}\left(\mathcal{T}, \Sigma, \mathcal{I}_{c}\right)$, which guarantees that $\tau$ is realized in $\mathcal{I}_{c}$ and that $a_{\tau}$ exists. We also note that each $w \in \Delta^{\mathcal{J}}$ is such that type $(w, \mathcal{J})=\operatorname{tail}(w)$, and, in particular, for fringe individuals from $a \in \Delta^{\mathcal{I}_{c}}$, it is the case that type $\left(a, \mathcal{I}_{c}\right)=\operatorname{type}(a, \mathcal{J})=\operatorname{tail}(a)$.

It is left to prove that $\mathcal{J}$ is an extension of $\mathcal{I}_{c}$, and a model of $\mathcal{K}$. For the former, we need to show that $\mathcal{J}$ satisfies the following conditions:

(i) $\Delta^{\mathcal{I}_{c}} \subseteq \Delta^{\mathcal{J}}$,

(ii) $A^{\mathcal{J}} \cap \Delta^{\mathcal{I}_{c}}=A^{\mathcal{I}_{c}}$,

(iii) $r^{\mathcal{J}} \cap\left(\Delta^{\mathcal{I}_{c}} \times \Delta^{\mathcal{I}_{c}}\right)=r^{\mathcal{I}_{c}}$, and

(iv) $q^{\mathcal{J}}=q^{\mathcal{I}_{c}}$ for all $q \in \Sigma$.

Clearly, $\Delta^{\mathcal{I}_{c}} \subseteq \Delta^{\mathcal{J}}$ since for all fringe individuals $a \in \Delta^{\mathcal{I}_{c}}, w=a$ is a run in $\operatorname{frn}\left(\mathcal{I}_{c}, \operatorname{str}\right)=\Delta^{\mathcal{J}}$, while for the individuals occurring in $\mathcal{K}$ the claim holds by construction and since $a^{\mathcal{I}_{c}}=a$ by the definition of a core. Next, for (ii) and (iii) we observe that, by construction of $\mathcal{J}, p^{\mathcal{I}_{c}} \subseteq p^{\mathcal{J}}$ for all role names $p$ and $A^{\mathcal{I}_{c}} \subseteq A^{\mathcal{J}}$ for all concept names $A$. That no other individual or fringe individual is added to $A$ is ensured by construction of $\operatorname{frn}\left(\mathcal{I}_{c}, \operatorname{str}\right)$, and the fact that $\operatorname{tail}(a)=\operatorname{type}\left(a, \mathcal{I}_{c}\right)=$ type $(a, \mathcal{J})$. Moreover, for any other pair $\left(w_{1}, w_{2}\right)$ added to $p^{\mathcal{J}}$, we have that at least one $w_{i}$ in the pair is of the form $a \ldots \alpha_{i} \tau_{i}$ for some $\tau_{i}$, with $i \geq 1$, that is not a c-type, and thus $w_{i} \notin \Delta^{\mathcal{I}_{c}}$. The latter is ensured by construction of $\mathcal{J}$ and the fact that runs of the form $a \alpha_{1} \tau_{1}$, where $\tau_{1}$ is a c-type, are not allowed in $\operatorname{crn}\left(\mathcal{I}_{c}, s t r\right)$.

Finally we show that $q^{\mathcal{J}}=q^{\mathcal{I}_{c}}$ for all role names and concept names $q \in \Sigma$. More precisely we need to show that:

(a) for all $A \in \Sigma \cap \mathrm{N}_{\mathrm{C}}, e \in A^{\mathcal{J}}$ iff $e \in A^{\mathcal{I}_{c}}$, and

(b) for all $r \in \Sigma \cap \mathrm{N}_{\mathrm{R}},\left(e_{1}, e_{2}\right) \in r^{\mathcal{J}}$ iff $r\left(e_{1}, e_{2}\right) \in r^{\mathcal{I}_{c}}$.

By (ii) and (iii) we know that (a) and (b) hold for all $e$ in $\Delta^{\mathcal{I}_{c}}$. It is thus left to show that no other domain object is added to any of the closed concept names or closed role names in $\mathcal{J}$. First, assume $w \in \Delta^{\mathcal{J}} \backslash \Delta^{\mathcal{I}_{c}}$ is an object outside the core, that is, $w$ is of the form $w=a \alpha_{1} \tau_{1} \ldots \alpha_{\ell} \tau_{\ell}$ with $\ell \geq 1$, and $\tau_{\ell}$ is not a c-type. Thus, $A \in \Sigma$ implies $A \notin \tau_{\ell}$, and thus by construction, $w \notin A^{\mathcal{J}}$. For a role name $r \in \Sigma$, that no domain object other than individuals belong to $r$ in $\mathcal{J}$ is ensured by the fact that inclusions of the form $\alpha_{i}=A \sqsubseteq \exists r_{i} . A^{\prime} \in \mathcal{T}$ with $r_{i} \in \mathcal{T} \Sigma$, that is $r_{i} \sqsubseteq_{\mathcal{T}}^{*} r$ with $r^{(-)} \in \Sigma$, do not appear in any run in crn or 
frn. Note that by definition a type containing $A$ is a c-type, and runs in $c r n$ cannot continue after a c-type. Moreover, runs start from fringe individuals which, by definition of a core, cannot realize c-types.

It remains to prove that $\mathcal{J}$ models $\mathcal{K}$, that is $\mathcal{J} \models_{\Sigma} \mathcal{A}$ and $\mathcal{J} \models \mathcal{T}$. First, $\mathcal{J} \models_{\Sigma} \mathcal{A}$ is a direct consequence of the fact that $\mathcal{I}_{c} \models_{\Sigma} \mathcal{A}$ and that $\mathcal{J}$ is an extension of $\mathcal{I}_{c}$. To prove $\mathcal{J} \models \mathcal{T}$, we show that $\mathcal{J}$ satisfies all inclusions of the forms $(\mathbf{N} 1),(\mathbf{N 2}),(\mathbf{N} 3)$, and $(\mathbf{N} 4)$ in $\mathcal{T}$. To improve readability, this more technical part of the proof is given in the appendix.

For " $\Leftarrow$ ", assume an arbitrary model $\mathcal{J}$ of $\mathcal{K}$ that is an extension of $\mathcal{I}_{c}$. We show that we can extract from it a non-losing strategy for Bob. First, let $T$ be the set of all the types realized in $\mathcal{J}$, and observe that $T \subseteq \mathrm{LC}\left(\mathcal{T}, \Sigma, \mathcal{I}_{c}\right)$ and that for all $a \in \mathrm{N}_{\mathrm{l}}(\mathcal{K})$, type $(a, \mathcal{J}) \in T$.

We define a strategy str as follows:

(*) For each $\tau \in T$ that is not a c-type and each $A \sqsubseteq \exists r . A^{\prime} \in \mathcal{T}$, with $A \in \tau$, we $\operatorname{set} \operatorname{str}\left(\tau, A \sqsubseteq \exists r . A^{\prime}\right)=\tau^{\prime}$ for an arbitrarily chosen $\tau^{\prime} \in T$ that satisfies $(\mathrm{C} 1)$ and $(\mathrm{C} 2)$.

The preceding is possible because $\mathcal{J}$ is a model, hence it satisfies all existential and universal inclusions in $\mathcal{T}$, and because all types realized in $\mathcal{J}$ are contained in $T$. More precisely, assume an arbitrary type $\tau \in T$ that is not a c-type, let $c$ be an arbitrary object that realizes $\tau$ in $\mathcal{J}$ and assume an inclusion $A \sqsubseteq \exists r . A^{\prime} \in \mathcal{T}$. We fix $\operatorname{str}\left(\tau, A \sqsubseteq \exists r . A^{\prime}\right)$ to be the type $\tau^{\prime}$ that is realized by $c^{\prime}$, for an arbitrary object $c^{\prime} \in \Delta^{\overline{\mathcal{J}}}$ that satisfies $c^{\prime} \in A^{\prime \mathcal{J}}$ and $\left(c, c^{\prime}\right) \in r^{\mathcal{J}}$. The existence of such a type $\tau^{\prime}$ is guaranteed from the fact that $c \in A^{\mathcal{J}}$ and that $\mathcal{J}$ satisfies $A \sqsubseteq \exists r . A^{\prime}$. Clearly, $A^{\prime} \in \tau^{\prime}$, that is $\tau_{i}$ satisfies (C1). In addition, $\mathcal{J}$ being a model of $\mathcal{K}$, and in particular satisfying all universal and role inclusions of $\mathcal{K}$, guarantees that $\tau^{\prime}$ also satisfies (C2). More precisely, assume an arbitrary inclusion $A_{1} \sqsubseteq \forall s . A_{2} \in \mathcal{T}$ such that $r \sqsubseteq_{\mathcal{T}}^{*} s$ and $A_{1} \in \tau$. We have to show that $A_{2} \in \tau^{\prime}$ is the case. First, $c \in A_{1}^{\mathcal{J}}$ and $\left(c, c^{\prime}\right) \in s^{\mathcal{J}}$ are direct consequences of the assumption that $\tau=\operatorname{type}(c, \mathcal{J})$ and the fact that $\mathcal{J} \models r \sqsubseteq s$ holds. The latter together with the fact that $\mathcal{J} \models A_{1} \sqsubseteq \forall s . A_{2}$ imply that $c^{\prime} \in A_{2}^{\mathcal{J}}$. It thus follows $A_{2} \in \tau^{\prime}$ since $\tau^{\prime}=\operatorname{type}\left(c_{1}, \mathcal{J}\right)$. Similarly, assume an arbitrary inclusion $A_{1} \sqsubseteq \forall s . A_{2} \in \mathcal{T}$ such that $r^{-} \sqsubseteq_{\mathcal{T}}^{*} s$ and $A_{1} \in \tau^{\prime}$. It follows that $\left(c^{\prime}, c\right) \in s^{\mathcal{J}}$ and $c^{\prime} \in A_{1}^{\mathcal{J}}$. Consequently, $c \in A_{2}^{\mathcal{J}}$ and therefore $A_{2} \in \tau$ since type $(c, \mathcal{J})=\tau$.

Finally, one can see that str is a non-losing strategy for Bob on $\mathcal{I}_{c}$. For every run $w$ that follows str, the fact that $T \subseteq \mathrm{LC}\left(\mathcal{T}, \Sigma, \mathcal{I}_{c}\right)$ guarantees that $\operatorname{tail}(w) \in \mathrm{LC}\left(\mathcal{T}, \Sigma, \mathcal{I}_{c}\right)$. Condition $\left(^{*}\right)$ ensures that $\operatorname{str}(\operatorname{tail}(w), A \sqsubseteq$ $\left.\exists r . A^{\prime}\right)$ is defined for every $A \sqsubseteq \exists r . A^{\prime} \in \mathcal{T}$ with $A \in \operatorname{tail}(w)$.

By putting Lemma 3 and Proposition 2 together, we obtain the main result of this section, stated in the following theorem.

Theorem 2. Assume a c-safe $O M Q Q=(\mathcal{T}, \Sigma, \mathfrak{q}(\vec{x}))$ and let $\mathcal{K}=(\mathcal{T}, \Sigma, \mathcal{A})$ be a KB where $\mathcal{A}$ is an $A B$ Box over the concept and role names occurring in $\mathcal{T}$. Then $\mathcal{K} \not \models \mathfrak{q}(\vec{a})$ for a tuple of individuals $\vec{a}$ occurring in $\mathcal{K}$ iff there exists a core $\mathcal{I}_{c}$ for $\mathcal{K}$ such that

(1) $\mathcal{I}_{c} \not \models \mathfrak{q}(\vec{a})$, and

(2) there is a non-losing strategy for Bob on $\mathcal{I}_{c}$.

Example 4. Consider again our running example and consider the assertion $r_{1}(a, a)$. We can see that $\mathcal{K} \not$ $r_{1}(a, a)$. This is witnessed by the core $\mathcal{I}_{c}^{1}$, which (unlike $\mathcal{I}_{c}^{2}$ ) does not satisfy $r_{1}(a, a)$, and the non-losing strategy for Bob on $\mathcal{I}_{c}^{1}$ presented above.

\section{The Marking Algorithm}

In this section, to decide whether Bob has a non-losing strategy on a given core we use the type elimination procedure Mark in Algorithm 1, which marks (or eliminates) all types from which Sam has a strategy to defeat Bob. It takes as input the TBox $\mathcal{T}$, the set $\Sigma \subseteq \mathrm{N}_{\mathrm{C}} \cup \mathrm{N}_{\mathrm{R}}$, and a core $\mathcal{I}_{c}$ for some $\mathrm{KB} \mathcal{K}=(\mathcal{T}, \Sigma, \mathcal{A})$. The algorithm starts by building the set $N$ of all possible types over $\mathcal{T}$, and then it marks types that are not good choices for Bob. In steps $\left(\mathrm{M}_{\mathrm{N} 1}\right)$ and $\left(\mathrm{M}_{\Sigma}\right)$ the algorithm respectively marks in $N$ all types that 


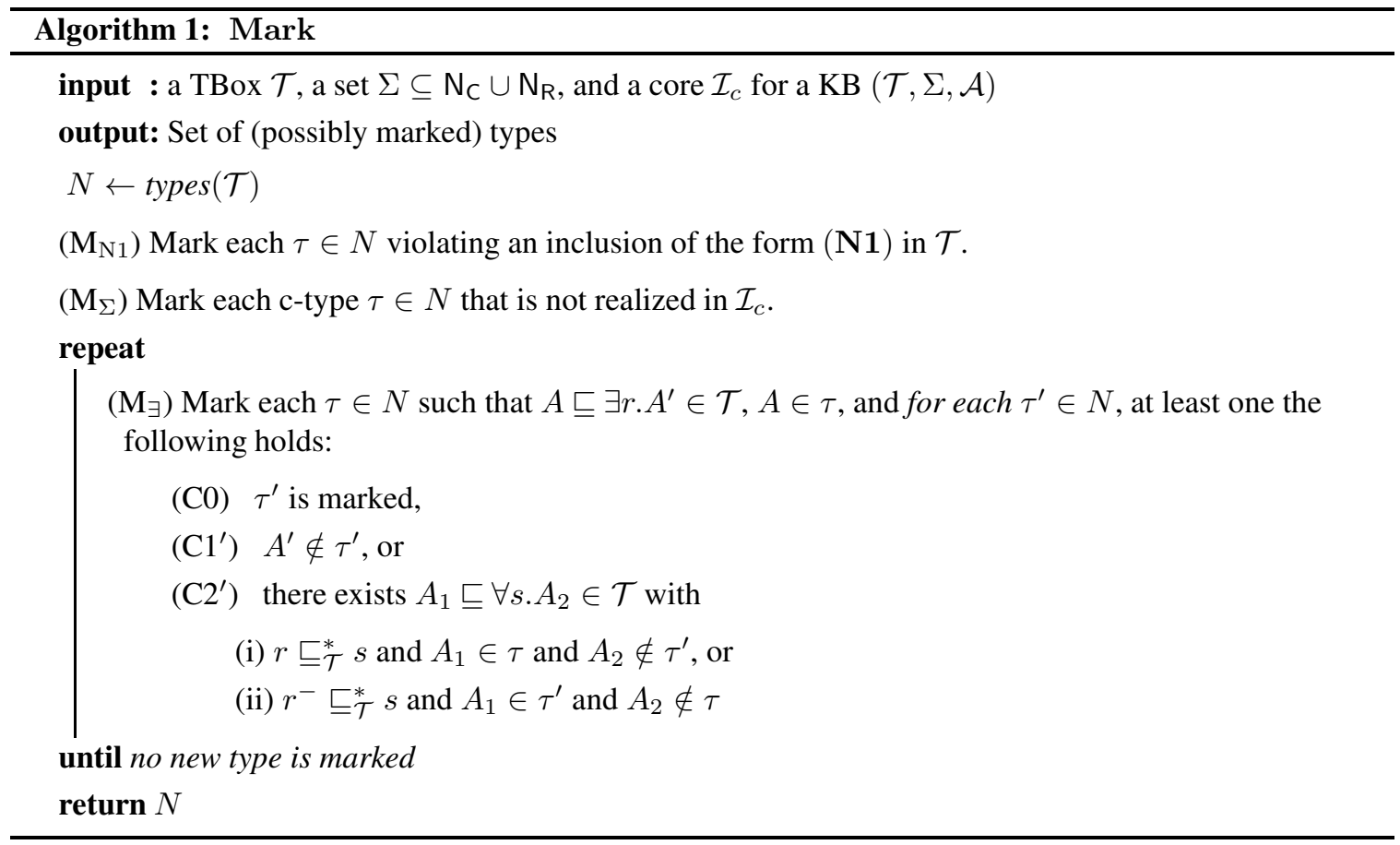

violate the conditions $\left(\mathrm{LC}_{\mathrm{N} 1}\right)$ or $\left(\mathrm{LC}_{\Sigma}\right)$; Bob is not allowed to choose those types. Then, in the loop, $\left(\mathrm{M}_{\exists}\right)$ exhaustively marks types $\tau$ that, if picked by Bob, would allow Sam to pick an inclusion $A \sqsubseteq \exists r$. $A^{\prime}$ for which Bob cannot reply with any $\tau^{\prime}$.

The correspondence between types that are marked by the algorithm, and those that may occur in runs that follow a non-losing strategy is provided in the following proposition.

Proposition 3. Consider a $K B \mathcal{K}=(\mathcal{T}, \Sigma, \mathcal{A})$, a core $\mathcal{I}_{c}$ for $\mathcal{K}$, and a non-losing strategy str for Bob on $\mathcal{I}_{c}$. For every $\tau \in$ types $(\mathcal{T})$, if $\tau$ is marked by $\operatorname{Mark}\left(\mathcal{T}, \Sigma, \mathcal{I}_{c}\right)$, then there is no run $w$ following str with $\operatorname{tail}(w)=\tau$.

Proof. Let str be a non-losing strategy on $\mathcal{I}_{c}$. Consider an arbitrary $\tau$ marked by $\operatorname{Mark}\left(\mathcal{T}, \Sigma, \mathcal{I}_{c}\right)$. We denote by $N_{0}$ the set of types that are marked before the loop, by $\left(\mathrm{M}_{\mathrm{N} 1}\right)$, or $\left(\mathrm{M}_{\Sigma}\right)$; and we denote by $N_{i}$ the types that are marked in the $i$-th iteration of the loop. We show by induction on $k$ that, if $\tau \in N_{k}$, then there is no run $w$ following $\operatorname{str}$ with $\operatorname{tail}(w)=\tau$.

For the base case, $k=0, \tau \in N_{0}$ implies that $\tau$ is marked by one of $\left(\mathrm{M}_{\mathrm{N} 1}\right)$, or $\left(\mathrm{M}_{\Sigma}\right)$. Then $\tau$ does not satisfy $\left(\mathrm{LC}_{\mathrm{N} 1}\right)$ or $\left(\mathrm{LC}_{\Sigma}\right)$, which implies that $\tau \notin \mathrm{LC}(\mathcal{T}, \Sigma, \mathcal{I})$ and hence there cannot exist a run $w$ with $\operatorname{tail}(w)=\tau$, since this would contradict the definition of non-losing str.

For the inductive case, let $\tau \in N_{k}$. Let $\alpha=A \sqsubseteq \exists r$. $A^{\prime} \in T$ with $A \in \tau$ be the axiom chosen for $\left(\mathrm{M}_{\exists}\right)$ that results in the marking of $\tau$. Towards a contradiction, assume there exists a run $w$ that follows str and $\operatorname{tail}(w)=\tau$. By definition of non-losing strategies, $\operatorname{str}(\tau, \alpha)=\tau^{\prime}$ must be defined, and it must be such that:

$(\star) \quad \tau^{\prime} \in \mathrm{LC}\left(\mathcal{T}, \Sigma, \mathcal{I}_{c}\right), A^{\prime} \in \tau^{\prime}$, and for all inclusions $A_{1} \sqsubseteq \forall s . A_{2} \in \mathcal{T}$ :

- if $r \sqsubseteq \mathcal{T}^{*} s$ and $A_{1} \in \tau$ then $A_{2} \in \tau^{\prime}$,

- if $r^{-} \sqsubseteq_{\mathcal{T}}^{*} s$ and $A_{1} \in \tau^{\prime}$ then $A_{2} \in \tau$.

Then, since $w$ is a run that follows $\operatorname{str}$, and $\operatorname{str}(\tau, \alpha)=\tau^{\prime}$, then $w^{\prime}=w \alpha \tau^{\prime}$ is also a run that follows str. However, $(\star)$ implies that neither $\left(\mathrm{C}^{\prime}\right)$ nor $\left(\mathrm{C}^{\prime}\right)$ hold for $\tau^{\prime}$. Hence, since $\tau$ gets marked by $\left(\mathrm{M}_{\exists}\right)$, then $(\mathrm{C} 0)$ must hold, that is, $\tau^{\prime} \in N_{j}$ for some $j<k$. The latter together with the induction hypothesis imply that $\tau^{\prime}$ cannot be the tail of a run that follows str, and thus $w^{\prime}=w \alpha \tau^{\prime}$ cannot be a run that follows str, which is a contradiction. 
Now we can formally establish how the marking algorithm allows us to verify the existence of a nonlosing strategy for Bob on a given core.

Theorem 3. Consider a $K B \mathcal{K}=(\mathcal{T}, \Sigma, \mathcal{A})$, and a core $\mathcal{I}_{c}$ for $\mathcal{K}$. Then Bob has a non-losing strategy on $\mathcal{I}_{c}$ iff there is no fringe individual $c^{\alpha}$ in $\mathcal{I}_{c}$ whose type type $\left(c^{\alpha}, \mathcal{I}_{c}\right)$ is marked by $\operatorname{Mark}\left(\mathcal{T}, \Sigma, \mathcal{I}_{c}\right)$.

Proof. For the " $\Rightarrow$ " direction, we show that if there is some fringe individual $a$ in $\mathcal{I}_{c}$ that realizes a type that is marked by $\operatorname{Mark}\left(\mathcal{T}, \Sigma, \mathcal{I}_{c}\right)$, then there is no non-losing strategy for Bob on $\mathcal{I}_{c}$. Let $a$ be a fringe individual such that $\tau=\operatorname{type}\left(a, \mathcal{I}_{c}\right)$ is marked by $\operatorname{Mark}\left(\mathcal{T}, \Sigma, \mathcal{I}_{c}\right)$. Towards a contradiction, assume that a non-losing strategy str on $\mathcal{I}_{c}$ exists. But by definition, $a$ is a run that follows str, and since $\tau=\operatorname{tail}(a)$ is marked by $\operatorname{Mark}\left(\mathcal{T}, \Sigma, \mathcal{I}_{c}\right)$, by Proposition 3, $\tau$ cannot occur in a run that follows str, a contradiction.

For the " $\Leftarrow$ " direction, let $T$ be the set of all types that are not marked by the algorithm $\operatorname{Mark}\left(\mathcal{T}, \Sigma, \mathcal{I}_{c}\right)$; note that $T \subseteq \operatorname{LC}\left(\mathcal{T}, \Sigma, \mathcal{I}_{c}\right)$. Assume that type $\left(a, \mathcal{I}_{c}\right) \in T$ for every fringe individual $a$ in $\mathcal{I}_{c}$.

Then we can build a strategy str on $\mathcal{I}_{c}$ as follows.

(*) For each pair of $\tau \in T$ that is not a c-type and $\alpha=A \sqsubseteq \exists r . A^{\prime} \in \mathcal{T}$, with $A \in \tau$, we set $\operatorname{str}(\tau, \alpha)$ to an arbitrary $\tau^{\prime} \in T$ that satisfies $(\mathrm{C} 1)$ and $(\mathrm{C} 2)$.

Note that such a construction of str is possible, since a suitable $\tau^{\prime}$ always exists. If this were not the case for some pair then $\tau$ would clearly be marked by the algorithm at some iteration of $\left(\mathrm{M}_{\exists}\right)$.

We claim that str is a non-losing strategy on $\mathcal{I}_{c}$. Towards a contradiction, assume it is not, and let $w$ with $\operatorname{tail}(w)=\tau$ be a run that follows str. Then either $\tau \notin \operatorname{LC}\left(\mathcal{T}, \Sigma, \mathcal{I}_{c}\right)$, or $\tau$ is not a c-type and there exists some inclusion $\alpha=A \sqsubseteq \exists r . A^{\prime} \in \mathcal{T}$, with $A \in \tau$, and $\operatorname{str}(\tau, \alpha)$ is not defined. The former contradicts the fact that $\tau \in T$ and thus $T \subseteq \operatorname{LC}\left(\mathcal{T}, \Sigma, \mathcal{I}_{c}\right)$. The latter contradicts the existence of $\tau^{\prime}$ argued above. Hence, str is a non-losing strategy for Bob on $\mathcal{I}_{c}$.

Example 5. Consider again our running example. Four concept names and a nominal occur in $\mathrm{N}_{\mathrm{C}}^{+}(\mathcal{T})$, namely $A_{1}, A_{2}, A_{3}, A_{4}$, and $\{c\}$. Consequently, there are $2^{5}=32$ possible types over $\mathcal{T}$. The algorithm marks the following types (recall that we omit $T$ to avoid clutter):

- Every type containing $A_{2}$ but not $A_{3}$ or $A_{4}$ will be marked by $\left(\mathrm{M}_{\mathrm{N} 1}\right)$.

- All types that contain $A_{1}$ or $A_{4}$ other than $\left\{A_{1}, A_{4}\right\}$ and $\left\{A_{1}, A_{3}\right\}$, and all types that contain $\{c\}$ except for $\{\{c\}\}$ are marked by $\left(\mathrm{M}_{\Sigma}\right)$.

Of the still unmarked types \{\}$,\left\{A_{3}\right\}$, and $\left\{A_{2}, A_{3}\right\},\left\{A_{1}, A_{4}\right\},\left\{A_{1}, A_{3}\right\}$, and $\{\{c\}\}$, none gets marked in the loop, so they all remain unmarked.

\section{Rewriting into Datalog with Negation}

In this section, we provide a rewriting of a given c-safe ontology-mediated query $Q$ into DATALOG extended with negation, under the stable model semantics. The program in essence implements the marking algorithm from Section 5 , and the output of the program (in terms of the so-called skeptical entailment) corresponds to the certain answer to $Q$ over any input ABox. We later show that in the absence of closed predicates we can provide a rewriting into a disjunctive DATALOG program without negation. We start by recalling the relevant variants of DATALOG.

\section{DATALOG with Disjunction and Negation}

We assume countably infinite sets $N_{P}$ and $N_{V}$ of predicate symbols (each with an associated arity) and variables, respectively. We further assume that $\mathrm{N}_{\mathrm{C}} \cup \mathrm{N}_{\mathrm{R}} \subseteq \mathrm{N}_{\mathrm{P}}$ with each $A \in \mathrm{N}_{\mathrm{C}}$ being unary, and each $r \in \mathrm{N}_{\mathrm{R}}$ being binary. An atom is an expression of the form $R\left(t_{1}, \ldots, t_{n}\right)$, where $\left\{t_{1}, \ldots, t_{n}\right\} \subseteq \mathrm{N}_{\mathrm{I}} \cup \mathrm{N}_{\mathrm{V}}$, and $R$ is an $n$-ary relation symbol. A negated atom is an expression of the form not $\alpha$, where $\alpha$ is an atom. 
A (negated) atom is ground if it contains no variables, that is, $\left\{t_{1}, \ldots, t_{n}\right\} \subseteq \mathrm{N}_{\mathrm{I}}$. A rule $\rho$ is an expression of the form

$$
h_{1} \vee \ldots \vee h_{n} \leftarrow b_{1}, \ldots, b_{k}
$$

where $n, k \geq 0, H=\left\{h_{1}, \ldots, h_{n}\right\}$ is a set of atoms, called the head of $\rho$, and $B=\left\{b_{1}, \ldots, b_{k}\right\}$ is a set of possibly negated atoms, called the body of $\rho$. Each variable that occurs in $\rho$ must also occur in a (non-negated) atom in the body of $\rho$. If $|H|>1$, then we call $\rho$ a disjunctive rule. If negated atoms do not occur in $\rho$, then $\rho$ is positive. We say a rule $\rho$ is a constraint if $H=\emptyset$, that is if $\rho$ is of the form $\leftarrow b_{1}, \ldots, b_{k}$. Rules of the form $h \leftarrow$ (known as facts) are simply identified with the atom $h$, thus ABox assertions are valid facts in our syntax. For a role name $p$, we may use $p^{-}\left(t_{1}, t_{2}\right)$ to denote the atom $p\left(t_{2}, t_{1}\right)$. A program is any finite set $P$ of rules. If no disjunctive rules occur in $P$, we call $P$ a non-disjunctive program. Unless specified otherwise, we consider non-disjunctive programs by default. If all rules in $P$ are positive, then $P$ is also called positive. We use ground $(P)$ to denote the grounding of $P$, i.e. the variable-free program that is obtained from $P$ by applying on its rules all the possible substitutions of variables by individuals of $P$.

A (Herbrand) interpretation $I$, also called a database, is any finite set of variable-free (or ground) atoms. We assume a binary built-in inequality predicate $\neq$ with a natural meaning: in any interpretation $I, a \neq b \in I$ iff (i) $a \neq b$, and (ii) both $a, b$ occur in some atoms $R_{1}\left(\vec{t}_{1}\right), R_{2}\left(\vec{t}_{2}\right) \in I$, where neither of $R_{1}, R_{2}$ is the inequality predicate.

An interpretation $I$ is a model of a positive program $P$ if $\left\{b_{1}, \ldots, b_{k}\right\} \subseteq I$ implies $I \cap\left\{h_{1}, \ldots, h_{n}\right\} \neq \emptyset$ for all rules $h_{1} \vee \ldots \vee h_{n} \leftarrow b_{1}, \ldots, b_{k}$ in ground $(P)$. We say an interpretation $I$ is a minimal model of a positive program $P$ if $I$ is a model of $P$, and there is no $J \subsetneq I$ that is a model of $P$.

The GL-reduct of a program $P$ with respect to an interpretation $I$ is the program $P^{I}$ that is obtained from ground $(P)$ in two steps [25]:

(i) deleting every rule that has not $\alpha$ in the body with $\alpha \in I$, and

(ii) deleting all negated atoms in the remaining rules.

An interpretation $I$ is a stable model (also known as an answer set) of a program $P$ if $I$ is a minimal model of $P^{I}$.

We call query a pair $(P, q)$ of a program $P$ and a predicate symbol $q$ occurring in $P$. A tuple $\vec{a}$ of constants is a certain answer to $(P, q)$ over a database $I$ if $q(\vec{a}) \in J$ for all stable models $J$ of $P \cup I$; the set of all such $\vec{a}$ is denoted cert $((P, q), I)$. A ground atom $q(\vec{a})$ is entailed from a program $P$ and a database $I$, written $(P, I) \models q(\vec{a})$, if $\vec{a}$ is a certain answer to $(P, q)$ over $I$.

\section{Rewriting OMQs}

In this section, for a given c-safe ontology-mediated query $Q=(\mathcal{T}, \Sigma, \mathfrak{q})$, where $\mathcal{T}$ is an $\mathcal{A L C H O I}$ TBox, we build a query $\left(P_{Q}, q\right)$, where $P_{Q}$ is a DATALOG program with negation, such that for every ABox $\mathcal{A}$ over the concept and role names occurring in $\mathcal{T}$ and for every tuple of constants $\vec{a}$, we have:

$$
(\mathcal{T}, \Sigma, \mathcal{A}) \models \mathfrak{q}(\vec{a}) \text { iff } \vec{a} \text { is a certain answer to }\left(P_{Q}, q\right) \text { over } \mathcal{A} \text {. }
$$

We later show that in case $\Sigma=\emptyset$, we can obtain a positive disjunctive program, which might employ the inequality predicate $\neq$. If additionally there are no nominals in $\mathcal{T}$, then $\neq$ is not necessary. Crucially, in all cases $\left(P_{Q}, q\right)$ can be built in polynomial time, and is independent of any input ABox.

The main challenge to obtain the desired program $P_{Q}$ is to build a program whose stable models correspond to the cores that can be extended into a model of $(\mathcal{T}, \Sigma, \mathcal{A})$, for any input $\mathrm{ABox} \mathcal{A}$. More precisely:

Proposition 4. Let $\mathcal{T}$ be a TBox, let $\Sigma$ be a set of closed predicates, and let $\mathcal{A}$ be an ABox over the concept and role names occurring in $\mathcal{T}$. Then we can build in polynomial time a program $P_{Q}^{*}$ such that the following hold:

- If $\mathcal{I}_{c}$ is a core for $(\mathcal{T}, \Sigma, \mathcal{A})$ that can be extended into a model of $(\mathcal{T}, \Sigma, \mathcal{A})$, then there is a stable model I of $\mathcal{A} \cup P_{Q}^{*}$ such that $(i) A(b) \in I$ iff $b \in A^{\mathcal{I}_{c}}$ for all concept names $A$, and (ii) $r(b, c) \in I$ iff $(b, c) \in r^{\mathcal{I}_{c}}$ for all role names $r$. 
- If I is a stable model of $\mathcal{A} \cup P_{Q}^{*}$, then there exists a core $\mathcal{I}_{c}^{I}$ for $(\mathcal{T}, \Sigma, \mathcal{A})$ that can be extended into a model of $(\mathcal{T}, \Sigma, \mathcal{A})$, and such that $(i) A(b) \in I$ iff $b \in A^{\mathcal{I}_{c}}$ for all concept names $A$, and (ii) $r(b, c) \in I$ iff $(b, c) \in r^{\mathcal{I}_{c}}$ for all role names $r$.

Before proving Proposition 4, we state our main result on rewritability. It is a direct consequence of Proposition 4 and the previously presented characterization of OMQ answering.

Theorem 4. For a c-acyclic $O M Q Q=(\mathcal{T}, \Sigma, \mathfrak{q})$, where $\mathcal{T}$ is an $\mathcal{A} \mathcal{L C H O I}$ TBox, we can build in polynomial time a query $\left(P_{Q}, q\right)$, where $P_{Q}$ is a DATALOG program with stable negation, such that $\operatorname{cert}(Q, \mathcal{A})=$ $\operatorname{cert}\left(\left(P_{Q}, q\right), \mathcal{A}\right)$ for any given $A B o x \mathcal{A}$ over the concept and role names occurring in $\mathcal{T}$.

Proof. This is an immediate consequence of Proposition 4 together with Lemma2, and Theorems 2 and 3 To obtain a polynomial time rewriting from a c-safe OMQ $Q=(\mathcal{T}, \Sigma, \mathfrak{q}(\vec{x}))$, we simply need to compute $P_{Q}^{*}$, pick a fresh predicate $q$ of the same arity as $\mathfrak{q}$, and add to $P_{Q}^{*}$ an additional rule that, for every database $D$, includes the atoms $q(\vec{c})$ in every stable model of $P_{Q}$ over $D$ whenever $\vec{c}$ is a certain answer of $Q$ over $\mathcal{A}$. We simply let $P_{Q}=P_{Q}^{*} \cup\{q(\vec{x}) \leftarrow \mathfrak{q}\}$.

We will dedicate the remainder of this section to prove Proposition 4 by building the program $P_{Q}^{*}$, which is defined as the union of three components:

$P_{c}$ is a set of rules that, given an input $\mathrm{ABox} \mathcal{A}$, non-deterministically generates all possible cores for $(\mathcal{T}, \Sigma, \mathcal{A})$.

$P_{M}$ is a set of rules that implement the type elimination algorithm presented in Section 5

$P_{T}$ is a set of rules that, relying on the marking done by $P_{M}$, filters out from the cores generated by $P_{c}$ those that cannot be extended into a model of $(\mathcal{T}, \Sigma, \mathcal{A})$, since they contain some fringe individual whose type is marked.

We construct $P_{c}, P_{M}$, and $P_{T}$ next.

\section{GENERATING THE CORES}

Let $\mathcal{T}$ be a TBox, let $\Sigma$ be a set of closed predicates, and let $\mathcal{A}$ be an ABox over the concept and role names occurring in $\mathcal{T}$. We start by describing $P_{c}$. Its main property is that, given an input $\mathrm{ABox} \mathcal{A}$, the stable models of $\mathcal{A} \cup P_{c}$ correspond to the cores for $(\mathcal{T}, \Sigma, \mathcal{A})$ : if $I$ is a stable model of $\mathcal{A} \cup P_{c}$, then we can extract a core for $(\mathcal{T}, \Sigma, \mathcal{A})$ from it, and conversely, if $\mathcal{I}_{c}$ is a core for $(\mathcal{T}, \Sigma, \mathcal{A})$, then we can define from it a stable model of $\mathcal{A} \cup P_{c}$.

(I) Collecting the individuals We start with rules to collect in the unary predicate ind all the individuals that occur in $\mathcal{T}$ or the input $\mathrm{ABox} \mathcal{A}$; this allows us to compute the set $\mathrm{N}_{\mathrm{I}}(\mathcal{K})$. For all nominals $\{a\}$ that occur in $\mathcal{T}$, for all $A \in \mathrm{N}_{\mathrm{C}}(\mathcal{T})$, and for all $p \in \mathrm{N}_{\mathrm{R}}(\mathcal{T})$, and we add to $P_{c}$ :

$$
\begin{array}{rlrl}
\operatorname{ind}(a) & \leftarrow & & \operatorname{ind}(x) \leftarrow p(x, y) \\
\operatorname{ind}(x) \leftarrow A(x) & & \operatorname{ind}(y) \leftarrow p(x, y)
\end{array}
$$

(II) Generating the cores Our next goal is to generate candidate cores. Recall that in cores, the set of original individuals of the knowledge bases can be expanded with a limited number of further elements. We need to simulate these elements, because they are not directly available to us. For every existential inclusion $\alpha$ in $\mathcal{T}$, let in ${ }^{\alpha}$ and out ${ }^{\alpha}$ be two fresh unary relations. We can now guess the domain of our candidate core. For all existential inclusions $\alpha$ in $\mathcal{T}$ we add the following rules:

$$
\begin{aligned}
\operatorname{in}^{\alpha}(x) & \leftarrow \operatorname{ind}(x), \text { not } \text { out }^{\alpha}(x) \\
\text { out }^{\alpha}(x) & \leftarrow \operatorname{ind}(x), \text { not } \text { in }^{\alpha}(x)
\end{aligned}
$$


Intuitively, the fact in ${ }^{\alpha}(c)$ means that the special constant $c^{\alpha}$ is included in the domain of the candidate core. To characterize the possible participation by the ordinary and the special constants in the relevant concept names, for each $A \in \mathrm{N}_{\mathrm{C}}(\mathcal{T}) \backslash \Sigma$, and all of existential inclusions $\alpha$ in $\mathcal{T}$, we take fresh unary predicates $\bar{A}$, $A^{\alpha}, \bar{A}^{\alpha}$, and add the following rules:

$$
\begin{aligned}
A(x) & \leftarrow \operatorname{ind}(x), \text { not } \bar{A}(x) \\
\bar{A}(x) & \leftarrow \operatorname{ind}(x), \text { not } A(x) \\
A^{\alpha}(x) & \leftarrow \operatorname{in}^{\alpha}(x), \text { not } \bar{A}^{\alpha}(x) \\
\bar{A}^{\alpha}(x) & \leftarrow \operatorname{in}^{\alpha}(x), \text { not } A^{\alpha}(x)
\end{aligned}
$$

Intuitively, an atom $A^{\alpha}(c)$ mean that $c^{\alpha}$ is the extension of $A$. To handle the participation in role names, for all $p \in N_{\mathrm{R}}(\mathcal{T}) \backslash \Sigma$ and existential inclusions $\alpha$ in $\mathcal{T}$, we take a fresh binary predicate $\bar{p}$, fresh unary predicates, $p_{\rightarrow}^{\alpha}, p_{\leftarrow}^{\alpha}, \bar{p}_{\rightarrow}^{\alpha}, \bar{p}_{\leftarrow}^{\alpha}$, and add the following rules:

$$
\begin{array}{rlrl}
p(x, y) & \leftarrow \operatorname{ind}(x), \operatorname{ind}(y), \operatorname{not} \bar{p}(x, y) & & \\
\bar{p}(x, y) & \leftarrow \operatorname{ind}(x), \operatorname{ind}(y), \text { not } p(x, y) & & \\
p_{D}^{\alpha}(x) & \leftarrow \operatorname{in}^{\alpha}(x), \operatorname{not} \bar{p}_{D}^{\alpha}(x) & D \in\{\rightarrow, \leftarrow\} \\
\bar{p}_{D}^{\alpha}(x) & \leftarrow \operatorname{in}^{\alpha}(x), \operatorname{not} p_{D}^{\alpha}(x) & D \in\{\rightarrow, \leftarrow\}
\end{array}
$$

Intuitively, an atom $p_{\rightarrow}^{\alpha}(c)$ (resp., $p_{\leftarrow}^{\alpha}(c)$ ) states that there is an $r$-link from $c$ to $c^{\alpha}$ (resp., from $c^{\alpha}$ to $c$ ). Note that the above rules are only for concept and role names not occurring in $\Sigma$. In this way, the extension of the closed predicates in $\Sigma$ is preserved in the stable models of $\mathcal{A} \cup P_{c}$, which is necessary to satisfy the conditions of the core as in Definition 7

(III) Validating cores We need to make sure that the structures generated by the rules in (I) and (II) satisfy the conditions (c3.1), (c3.2), (c3.3), (c3.4) and (c5) from Definition 7 The remaining conditions are trivially satisfied. Next we use $\hat{r}(x, y)$ to denote $p(x, y)$ if $r=p \in \mathrm{N}_{\mathbf{R}}$, and $p(y, x)$ if $r=p^{-}$for some $p \in \mathrm{N}_{\mathrm{R}}$. For an inverse role $r=p^{-}$with $p \in \mathrm{N}_{\mathrm{R}}, r_{\rightarrow}^{\alpha}$ denotes $p_{\leftarrow}^{\alpha}$.

To deal with (c3.1), we take an auxiliary fresh binary relation eq, and add to $P_{c}$ the rule eq $(x, x) \leftarrow \operatorname{ind}(x)$. Using $\{o\}(x)$ to denote eq $(x, a)$, and $\overline{\{o\}}(x)$ to denote $x \neq o$, we can add, for all inclusions $B_{1} \sqcap \cdots \sqcap B_{n} \sqsubseteq$ $B_{n+1} \sqcup \cdots \sqcup B_{k}$ in $\mathcal{T}$, and all existential inclusions $\alpha$ in $\mathcal{T}$, the following constraints:

$$
\begin{aligned}
& \leftarrow \operatorname{ind}(x), B_{1}(x), \ldots, B_{n}(x), \bar{B}_{n+1}(x), \ldots, \bar{B}_{k}(x) \\
& \leftarrow \operatorname{in}^{\alpha}(x), B_{1}^{\alpha}(x), \ldots, B_{n}^{\alpha}(x), \bar{B}_{n+1}^{\alpha}(x), \ldots, \bar{B}_{k}^{\alpha}(x)
\end{aligned}
$$

To ensure the satisfaction of (c3.2), for each $A \sqsubseteq \forall r . B \in \mathcal{T}$, and all existential inclusions $\alpha$ in $\mathcal{T}$ we add the following:

$$
\begin{aligned}
& \leftarrow A(x), \hat{r}(x, y), \operatorname{not} B(y) \\
& \leftarrow A(x), r_{\rightarrow}^{\alpha}(x), \text { not } B^{\alpha}(x) \\
& \leftarrow A^{\alpha}(x), r_{\leftarrow}^{\alpha}(x), \operatorname{not} B(x)
\end{aligned}
$$

To ensure (c3.3), for each $r \sqsubseteq s \in \mathcal{T}$, and all existential inclusions $\alpha$ in $\mathcal{T}$, we add the following:

$$
\begin{aligned}
& \leftarrow \hat{r}(x, y), \text { not } \hat{s}(x, y) \\
& \leftarrow r_{D}^{\alpha}(x), \operatorname{not} s_{D}^{\alpha}(x)
\end{aligned} \quad D \in\{\rightarrow, \leftarrow\}
$$

Let's deal with (c5). Assume an inclusion $A \sqsubseteq \exists r . B$ in $\mathcal{T}$. We take a fresh unary predicate $S$, and for all existential inclusions $\alpha$ in $\mathcal{T}$ we add the following:

$$
\begin{aligned}
S(x) & \leftarrow \hat{r}(x, y), B(y) \\
S(x) & \leftarrow r_{\rightarrow}^{\alpha_{1}}(x), B^{\alpha_{1}}(x) \\
& \leftarrow A(x), \text { not } S(x)
\end{aligned}
$$


Finally, to ensure (c3.4), for all existential inclusions $\alpha$ in $\mathcal{T}$, and all $A \sqsubseteq \exists r . A^{\prime} \in \mathcal{T}$ with $r \in_{\mathcal{T}} \Sigma$, we add the constraint $\leftarrow A^{\alpha}(x)$.

This finishes the construction of $P_{c}$, whose stable models are in one-to-one correspondence with the cores for $(\mathcal{T}, \Sigma, \mathcal{A})$. We explain next how $P_{M}$ and $P_{T}$ are built. In the explanations, we may blur the distinction between the stable models of $P_{c}$ and the actual cores.

\section{IMPLEMENTING THE ALGORITHM Mark}

We now move on to $P_{M}$, which implements the algorithm Mark from Section 5 To obtain a polynomially sized program, we need to use non-ground rules whose number of variables depends on the number of different concept names and nominals in $\mathrm{N}_{\mathrm{C}}^{+}(\mathcal{T})$. In a nutshell, in the grounding of $P_{M}$, types are represented as atoms of arity $\left|\mathrm{N}_{\mathrm{C}}^{+}(\mathcal{T})\right|$. The rules of $P_{M}$ generate these atoms and execute the marking algorithm on them: test for local consistency and for realization if required, iterate over all the types to search for suitable successors, etc. In general, the rules of $P_{M}$ use predicates with large arities, depending linearly on $\left|\mathrm{N}_{\mathrm{C}}^{+}(\mathcal{T})\right|$.

To represent types as ground atoms, we assume an arbitrary but fixed enumeration $B_{1}, \ldots, B_{k}$ of $\mathrm{N}_{\mathrm{C}}^{+}(\mathcal{T})$. $P_{M}$ relies heavily on the availability of at least two individuals. Here, we use a special pair of constants 0,1 that we add to the program using ground facts; note that our target language for rewriting OMQs is nonmonotonic disjunctive DATALOG with constants. The constants can be easily omitted if we assume the availability in the database of at least two distinct constants, which can be used in the place of 0,1 . We will come back to the role of these constants in Section 6

We now describe $P_{M}$. Below, the $k$-ary relation Type $=\{0,1\}^{k}$ stores the set of all types over $\mathcal{T}$. Naturally, a $k$-tuple $\left(b_{1}, \ldots, b_{k}\right) \in$ Type encodes the type $\left\{B_{i} \mid b_{i}=1,1 \leq i \leq k\right\} \cup\{\top\}$. The goal is to compute a $k$-ary relation Marked $\subseteq$ Type that contains precisely those types marked by the Mark algorithm. $P_{M}$ contains rules that compute Type, Marked, and some auxiliary relations.

(IV) A linear order over types We want the relation Type to contain all tuples in $\{0,1\}^{k}$. Of course, we could hard-code the list of these tuples, but there are exponentially many, thus the resulting program would not be polynomial. What we do instead is to encode a linear order over $\{0,1\}^{k}$, and starting from the first element, populate Type by adding successors. To this end, for every $1 \leq i \leq k$, we inductively define $i$-ary relations first ${ }^{i}$ and last ${ }^{i}$, and a $2 i$-ary relation next ${ }^{i}$, which will provide the first, the last and the successor elements from a linear order on $\{0,1\}^{i}$. In particular, given $\vec{u}, \vec{v} \in\{0,1\}^{i}$, the fact next ${ }^{i}(\vec{u}, \vec{v})$ will be true if $\vec{v}$ follows $\vec{u}$ in the ordering of $\{0,1\}^{i}$. The rules to populate next ${ }^{i}$ are quite standard (see, e.g., Theorem 4.5 in [17]). For the case $i=1$, we simply add the following facts:

$$
\text { first }^{1}(0) \leftarrow \quad \operatorname{last}^{1}(1) \leftarrow \quad \operatorname{next}^{1}(0,1) \leftarrow
$$

Then, for all $1<i \leq k-1$ we add the following rules:

$$
\begin{aligned}
\operatorname{next}^{i+1}(0, \vec{x}, 0, \vec{y}) & \leftarrow \operatorname{next}^{i}(\vec{x}, \vec{y}) \\
\operatorname{next}^{i+1}(1, \vec{x}, 1, \vec{y}) & \leftarrow \operatorname{next}^{i}(\vec{x}, \vec{y}) \\
\operatorname{next}^{i+1}(0, \vec{x}, 1, \vec{y}) & \leftarrow \operatorname{last}^{i}(\vec{x}), \operatorname{first}^{i}(\vec{y}) \\
\operatorname{first}^{i+1}(0, \vec{x}) & \leftarrow \operatorname{first}^{i}(\vec{x}) \\
\operatorname{last}^{i+1}(1, \vec{x}) & \leftarrow \operatorname{last}^{i}(\vec{x})
\end{aligned}
$$

We can now collect in the $k$-ary relation Type all types over $\mathcal{T}$ :

$$
\begin{aligned}
& \text { Type }(\vec{x}) \leftarrow \operatorname{first}^{k}(\vec{x}) \\
& \operatorname{Type}(\vec{y}) \leftarrow \operatorname{next}^{k}(\vec{x}, \vec{y})
\end{aligned}
$$

Type is the set $N$ of the Mark algorithm. In the rest of this section, we will write $\operatorname{vec}(\tau)$ to denote the bit vector encoding the type $\tau$. In a slight abuse of terminology, we may say that we mark $\tau$ to mean that we enforce Marked $(\operatorname{vec}(\tau))$ to hold, and if no confusion arises, we may interchange $\tau$ and $\operatorname{vec}(\tau)$. 
Below we describe rules that simulate the algorithm on this $N$. Recall that the algorithm runs on a given core $\mathcal{I}_{c}$. To implement the marking algorithm, we first mark all the types $\tau$ that do not satisfy the local consistency conditions $\operatorname{LC}\left(\mathcal{T}, \Sigma, \mathcal{I}_{c}\right)$. This is done with the rules described in items (V) to (VIII) below.

(V) Implementing Step $\left(\mathbf{M}_{\mathrm{N} 1}\right) \quad$ We use two auxiliary additional unary predicates $\mathrm{T}$ and $\mathrm{F}$ (intuitively, True and False), and add two facts to $P_{M}$ :

$$
\mathrm{F}(0) \leftarrow \quad \mathrm{T}(1) \leftarrow
$$

For a $k$-tuple of variables $\vec{x}$, we let $B \in \vec{x}$ denote the atom $\mathrm{T}\left(x_{j}\right)$, where $j$ is the index of $B$ in the enumeration of $\mathrm{N}_{\mathrm{C}}^{+}(\mathcal{T})$. Similarly, we let $B \notin \vec{x}$ denote the atom $\mathrm{F}\left(x_{j}\right)$, where $j$ is the index of $B$ in the enumeration.

The step $\left(\mathrm{M}_{\mathrm{N} 1}\right)$ of the algorithm, which marks types violating inclusions of type (N1), is implemented using the following rule in $P_{M}$, for every inclusion $B_{1} \sqcap \cdots \sqcap B_{n} \sqsubseteq B_{n+1} \sqcup \cdots \sqcup B_{k} \in \mathcal{T}$ :

$$
\operatorname{Marked}(\vec{x}) \leftarrow \operatorname{Type}(\vec{x}), B_{1} \in \vec{x}, \ldots, B_{n} \in \vec{x}, B_{n+1} \notin \vec{x}, \ldots, B_{k} \notin \vec{x}
$$

(VI) Collecting the realized types Our next goal is to compute a $k$-ary relation RealizedType such that, for each core $\mathcal{I}_{c}$, RealizedType will be populated with precisely the $\{0,1\}^{k}$-vectors that represent a type realized in $\mathcal{I}_{c}$ by some individual in $\mathrm{N}_{\mathrm{I}}(\mathcal{K})$. This relation will be useful in the rest of the marking algorithm.

To compute RealizedType, we use auxiliary $(i+1)$-ary relations hasType ${ }^{i}$ for all $0 \leq i \leq k$. We first put all the individuals into hasType ${ }^{0}$; intuitively, every individual has an 'empty' type up to the 0 -th position. Then we iteratively take an individual whose type has been stored up to the $(i-1)$-th position, and expand it to the $i$-th, using 0 or 1 according to whether or not the type that it realizes in $\mathcal{I}_{c}$ contains $B_{i}$. We first add to $P_{M}$ the rule:

$$
\operatorname{hasType}^{0}(x) \leftarrow \operatorname{ind}(x)
$$

and then, the following rules for all $1 \leq i \leq k$ :

$$
\begin{aligned}
\operatorname{hasType}^{i}(x, \vec{y}, 1) & \leftarrow \operatorname{hasType}^{i-1}(x, \vec{y}), B_{i}(x) & B_{i} \in \mathrm{N}_{\mathrm{C}} \\
\operatorname{hasType}^{i}(a, \vec{y}, 1) & \leftarrow \operatorname{hasType}^{i-1}(a, \vec{y}) & B_{i}=\{a\} \\
\operatorname{hasType}^{i}(x, \vec{y}, 0) & \leftarrow \operatorname{hasType}_{i}^{i-1}(x, \vec{y}), x \neq a & B_{i} \\
\operatorname{hasType}^{i}(x, \vec{y}, 0) & \leftarrow \operatorname{hasType}^{i-1}(x, \vec{y}), \bar{B}_{i}(x) & B_{i} \in \mathrm{N}_{\mathrm{C}} \backslash \Sigma \\
\operatorname{hasType}^{i}(x, \vec{y}, 0) & \leftarrow \operatorname{hasType}^{i-1}(x, \vec{y}), \text { not } B_{i}(x) & B_{i} \in \mathrm{N}_{\mathrm{C}} \cap \Sigma
\end{aligned}
$$

Intuitively, hasType ${ }^{k}(c, v e c(\tau))$ says that the individual $c$ realizes the type $\tau$. We can now project away the individuals and store in the relation RealizedType the set of all types realized in $\mathcal{I}_{c}$ :

$$
\text { RealizedType }(\vec{y}) \leftarrow \operatorname{hasType}^{k}(x, \vec{y})
$$

(VII) Implementing Step $\left(\mathbf{M}_{\Sigma}\right) \quad$ In this step, we mark all the non-realized types that are c-types. The following rules are added to $P_{M}$. First, we use a fresh $k$-ary predicate ClosedType to collect all the c-types. In particular, for each $B$ such that (i) $B$ is a nominal from $\mathcal{T}$, (ii) $B \in \mathrm{N}_{\mathrm{C}}(\mathcal{T}) \cap \Sigma$, or (iii) $\mathcal{T}$ contains an inclusion $B \sqsubseteq \exists r . A$ for some $A$ and $r \in_{\mathcal{T}} \Sigma$, we add:

$$
\text { ClosedType }(\vec{x}) \leftarrow \operatorname{Type}(\vec{x}), B \in \vec{x}
$$

We can now mark the c-types that are not realized, using a simple rule:

$$
\operatorname{Marked}(\vec{x}) \leftarrow \text { ClosedType }(\vec{x}), \text { not RealizedType }(\vec{x})
$$

The rules in (V) to (VII) mark, for a core $\mathcal{I}_{c}$, the types over $\mathcal{T}$ that do not satisfy the local consistency conditions $\operatorname{LC}\left(\mathcal{T}, \Sigma, \mathcal{I}_{c}\right)$. Now we move to the next step: marking the types for which there is no suitable successor type. 
(VIII) Implementing Step ( $\left.\mathbf{M}_{\exists}\right) \quad$ Consider an inclusion $\alpha=A \sqsubseteq \exists r . A^{\prime} \in \mathcal{T}$ with $r \notin \mathcal{T} \quad \Sigma$. Recall that we need to mark a type $\tau$ if $A \in \tau$, and for each type $\tau^{\prime} \in N$ at least one of (C0), (C1'), or (C2') holds. To this aim we use, for each such $\alpha$, an auxiliary $2 k$-ary relation MarkedOne ${ }_{\alpha}$ and collect, for each type $\tau$, all types $\tau^{\prime}$ that cannot be used to satisfy $\alpha$. That is, a pair $\left(\operatorname{vec}(\tau), \operatorname{vec}\left(\tau^{\prime}\right)\right)$ will be in MarkedOne $e_{\alpha}$ if $\tau^{\prime}$ is marked for $\tau$ by conditions ( $\mathrm{C} 0),\left(\mathrm{Cl}^{\prime}\right),\left(\mathrm{C}^{\prime}\right)$. Then we will iterate over our ordered list of types and test whether all $\tau^{\prime}$ are marked for $\tau$; in that case, we will mark $\tau$ if $A \in \tau$.

The following rules are added to $P_{M}$ for each $\alpha$ as above. We start with the rules for MarkedOne ${ }_{\alpha}$.

- For $(\mathrm{C} 0)$, we collect all pairs $\left(\operatorname{vec}(\tau), \operatorname{vec}\left(\tau^{\prime}\right)\right)$ where $\tau^{\prime}$ is marked:

$$
\text { MarkedOne }_{\alpha}(\vec{x}, \vec{y}) \leftarrow \operatorname{Type}(\vec{x}), \operatorname{Marked}(\vec{y})
$$

- For $\left(\mathrm{C1}^{\prime}\right)$, we collect pairs $\left(\operatorname{vec}(\tau), \operatorname{vec}\left(\tau^{\prime}\right)\right)$ with $A^{\prime} \notin \tau^{\prime}$ :

$$
\text { MarkedOne }_{\alpha}(\vec{x}, \vec{y}) \leftarrow \operatorname{Type}(\vec{x}), \operatorname{Type}(\vec{y}), A^{\prime} \notin \vec{y}
$$

- For $\left(\mathrm{C}^{\prime}\right)$, we proceed as follows.

- For all $A_{1} \sqsubseteq \forall s . A_{2} \in \mathcal{T}$ with $r \sqsubseteq_{\mathcal{T}}^{*} s$, we collect all $\left(\operatorname{vec}(\tau), \operatorname{vec}\left(\tau^{\prime}\right)\right)$ such that $A_{1} \in \tau$ and $A_{2} \notin \tau^{\prime}$ :

$$
\operatorname{MarkedOne}_{\alpha}(\vec{x}, \vec{y}) \leftarrow \operatorname{Type}(\vec{x}), \operatorname{Type}(\vec{y}), A_{1} \in \vec{x}, A_{2} \notin \vec{y}
$$

- For all $A_{1} \sqsubseteq \forall s . A_{2} \in \mathcal{T}$ with $r^{-} \sqsubseteq_{\mathcal{T}}^{*} s$, we collect all $\left(\operatorname{vec}(\tau), \operatorname{vec}\left(\tau^{\prime}\right)\right)$ such that $A_{1} \in \tau^{\prime}$ and $A_{2} \notin \tau$ :

$$
\text { MarkedOne }_{\alpha}(\vec{x}, \vec{y}) \leftarrow \operatorname{Type}(\vec{x}), \text { Type }(\vec{y}), A_{1} \in \vec{y}, A_{2} \notin \vec{x}
$$

Now we want to infer $\operatorname{Marked}(\operatorname{vec}(\tau))$ if $A$ is set to true in a type $\tau$, and $\operatorname{MarkedOne}_{\alpha}\left(\operatorname{vec}(\tau), \operatorname{vec}\left(\tau^{\prime}\right)\right)$ is true for all types $\tau^{\prime}$. To achieve this, we rely on another auxiliary $2 k$-ary relation MarkedUntil $\alpha_{\alpha}$ for each inclusion $\alpha$ :

$$
\begin{aligned}
& \operatorname{MarkedUntil}_{\alpha}(\vec{x}, \vec{z}) \leftarrow \operatorname{MarkedOne}_{\alpha}(\vec{x}, \vec{z}), \text { first }^{k}(\vec{z}) \\
& \operatorname{MarkedUntil}_{\alpha}(\vec{x}, \vec{u}) \leftarrow \operatorname{MarkedUntil}_{\alpha}(\vec{x}, \vec{z}), \operatorname{next}^{k}(\vec{z}, \vec{u}), \\
& \operatorname{MarkedOne}_{\alpha}(\vec{x}, \vec{u})
\end{aligned}
$$

Intuitively, with the above rules we traverse all types $\tau^{\prime}$ checking MarkedOne $e_{\alpha}$ for the pair $\left(\operatorname{vec}(\tau), \operatorname{vec}\left(\tau^{\prime}\right)\right)$. We mark $\tau$ if we reach the last $\tau^{\prime}$, and $A \in \tau$ :

$$
\operatorname{Marked}(\vec{x}) \leftarrow \operatorname{MarkedUntil}_{\alpha}(\vec{x}, \vec{z}), A \in \vec{x}, \text { last }^{k}(\vec{z})
$$

This completes the rules of $P_{M}$. Now we move to the construction of $P_{T}$ which essentially uses the marked type from $P_{M}$ to forbid the cores, generated by $P_{c}$, that cannot be extended into a model.

\section{FILTERING OUT CORES THAT CANNOT BE EXTENDED}

Finally, the program $P_{T}$ filters out from the cores generated by $P_{c}$ those that cannot be extended into a model of $(\mathcal{T}, \Sigma, \mathcal{A})$. More precisely, $P_{T}$ forbids a core $\mathcal{I}_{c}$ when a type realized in $\mathcal{I}_{c}$ is marked by the rules in $P_{M}$.

(IX) Forbidding marked types in the core For Theorem 3, we need to ensure that each type that is realized in $\mathcal{I}_{c}$ by a fringe individual is not marked by the algorithm Marked. For this we first compute a $k$-ary relation FringeType such that, for each core $\mathcal{I}_{c}$, FringeType will be populated with precisely the $\{0,1\}^{k_{-}}$ vectors that represent a type realized by a fringe individual in $\mathcal{I}_{c}$. 
To compute FringeType, we use auxiliary $(i+1)$-ary relations hasType ${ }_{\alpha}^{i}$ for all $0 \leq i \leq k$ and all existential inclusions $\alpha$ in $\mathcal{T}$. For each existential inclusion $\alpha$ in $\mathcal{T}$, and for all $1 \leq i \leq k$, we add to $P_{M}$ the following rules:

$$
\begin{aligned}
\operatorname{hasType}_{\alpha}^{0}(x) & \leftarrow \operatorname{ind}(x) & \\
\operatorname{hasType}_{\alpha}^{i}(x, \vec{y}, 1) & \leftarrow \operatorname{hasType}_{\alpha}^{i-1}(x, \vec{y}), B_{i}^{\alpha}(x) & B_{i} \in \mathrm{N}_{\mathrm{C}} \\
\operatorname{hasType}_{\alpha}^{i}(x, \vec{y}, 0) & \leftarrow \operatorname{hasType}_{\alpha}^{i-1}(x, \vec{y}) & B_{i}=\{a\} \\
\operatorname{hasType}_{\alpha}^{i}(x, \vec{y}, 0) & \leftarrow \operatorname{hasType}_{\alpha}^{i-1}(x, \vec{y}), \bar{B}_{i}^{\alpha}(x) & B_{i} \in \mathrm{N}_{\mathrm{C}} \backslash \Sigma
\end{aligned}
$$

Intuitively, hasType $\mathrm{e}_{\alpha}^{k}(c, \operatorname{vec}(\tau))$ says that the individual $c^{\alpha}$ realizes the type $\tau$. We can now project away the individuals and store in the relation FringeType the set of all types realized by fringe elements in $\mathcal{I}_{c}$. For all existential inclusions $\alpha$ in $\mathcal{T}$ we add:

$$
\text { FringeType }(\vec{y}) \leftarrow \operatorname{hasType}_{\alpha}^{k}(x, \vec{y})
$$

Finally, prohibiting marked types at fringe individuals is done by adding to $P_{T}$ the rule:

$$
\leftarrow \operatorname{Marked}(\vec{x}) \text {, FringeType }(\vec{x})
$$

This concludes the description of $P_{T}$, and hence of the program $P_{Q}^{*}=P_{c} \cup P_{M} \cup P_{T}$ described in Proposition 4

It remains to argue that $P_{Q}$ is of size polynomial in the size of $\mathcal{T}$. Indeed, $P_{c}$ is linearly bounded by the size of $\mathrm{N}_{\mathrm{C}}^{+}(\mathcal{T})$, the number of role names in $\mathrm{N}_{\mathrm{R}}(\mathcal{T})$ and the number of inclusions that appear in $\mathcal{T} ; P_{M}$ is bounded polynomially on the size of $\mathrm{N}_{\mathrm{C}}^{+}(\mathcal{T})$ (in particular the rules in (VII)) and in the number of inclusions in $\mathcal{T}$ (the rules in (VIII)); similarly as for $P_{M}, P_{T}$ is also bounded polynomially in the number of inclusions in $\mathcal{T}$.

\section{Complexity of evaluating the program}

In this section, for a given c-safe OMQ $Q=(\mathcal{T}, \Sigma, \mathfrak{q})$, we analyze the data and combined complexity of evaluating the program $P_{Q}$ for a given $\mathrm{ABox} \mathcal{A}$. The decision problem associated to answering a query $\left(P_{Q}, q\right)$ is analogous to that of OMQs defined in Section 3 , that is given a query $(P, q)$, where $P$ is a DATALOG program with negation under the stable model semantics, a (possibly empty) tuple of individuals $\vec{a}$, and an ABox $\mathcal{A}$, decide whether $\vec{a} \in \operatorname{cert}((P, q), \mathcal{A})$.

With our translation, we can obtain the following upper bounds. The bounds themselves (which are tight) are minor variations of results in the literature (e.g., [38]), and the aim of the proposition is to show the adequacy of our technique.

Proposition 5. Let $Q=(\mathcal{T}, \Sigma, \mathfrak{q})$ be a c-safe OMQ, where $\mathcal{T}$ is an $\mathcal{A} \mathcal{L C H O I}$ TBox. The problem to decide if $\vec{a}$ is a certain answer of $\left(P_{Q}, q\right)$ for a given $A B$ ox $\mathcal{A}$ over the concept and role names that occur in $\mathcal{T}$ is in EXPTIME w.r.t. combined complexity, and in coNP w.r.t. data complexity.

Proof. The coNP bound for data complexity follows easily since it is known that query answering for DATALOG programs with negation under the stable model semantics is coNP-complete in data complexity (see e.g., [17]). The result on the combined complexity does not follow directly from the complexity of query answering in DATALOG with negation, which is coNEXPTIME-complete [19]. Thus we need to argue more carefully about the shape of the program that results from our rewriting. In the program $P_{Q}$ some of the predicates have small arities, and the negation is used in a restricted way. As we shall see next, for these reasons our programs fall into a class of programs that can be evaluated in (deterministic) exponential time.

In the following, we say that a program $P$ defines a relation $R$, if $R$ appears in the head of a rule in $P$. The program $P_{Q}$ can be partitioned into programs $P_{1}, P_{2}, P_{3}$ as follows:

- $P_{1}$ consists of all rules in (I), (II), and (III). $P_{1}$ is a program with at most two variables in each rule. 
- $P_{2}$ consists of the rules in (VI), which define the relations hasType ${ }^{i}$ and the relation RealizedType.

- $P_{3}$ consists of the remaining rules.

Note that $P_{2}$ and $P_{3}$ do not define any relations used in $P_{1}$. The program $P_{2}$ only depends on $P_{1}$, that is, none of the relation symbols in $P_{2}$ is defined in $P_{3}$. The negative atoms of $P_{2}$ only involve relations that are only defined in $P_{1}$. Similarly, the negative atoms of $P_{3}$ only involve relations that are only defined by $P_{1} \cup P_{2}$. Assume a set $F$ of facts over the signature of $P_{1}$. Due to the above properties, the successful runs of the following non-deterministic procedure generate the set of all stable models of $P \cup F$ :

(S1) Compute a stable model $I_{1}$ of $P_{1} \cup F$.

(S2) Compute the least model $I_{2}$ of $I_{1} \cup P_{2}^{I_{1}}$. If $I_{2}$ does not exist due to a constraint violation, then return failure.

(S3) Compute the least model $I_{3}$ of $I_{2} \cup P_{2}^{I_{2}}$. Again, if $I_{3}$ does not exist, then return failure. Otherwise, output $I_{3}$.

Since $P_{1}$ has at most two variables in every rule, each stable model $I_{1}$ of $P_{1} \cup F$ is of polynomial size in the size of $P_{1} \cup F$, and the set of all such models can be traversed in polynomial space. For a given $I_{1}$, performing steps (S2) and (S3) is feasible in (deterministic) exponential time, because $P_{2}^{I_{1}}$ and the subsequent $P_{2}^{I_{2}}$ are ground disjunction-free positive programs of exponential size. It follows that computing the certain answers to $\left(P_{Q}, q\right)$ for any given $\mathrm{ABox} \mathcal{A}$ over the concept and role names of $\mathcal{T}$ requires only deterministic exponential time.

\section{Obtaining Positive Programs}

We now discuss the case of OMQs $Q=(\mathcal{T}, \Sigma, \mathfrak{q})$ without closed predicates, i.e. when $\Sigma=\emptyset$. We argue that in this restricted case, we can obtain a rewriting into a positive disjunctive program. In addition, if nominals are not present in $\mathcal{T}$, we do not even need the $\neq$ predicate. Towards this result, we observe that in case closed predicates are absent, we can simplify the notion of cores, and thus also the rules required for generating cores (in particular, the rules in (II) and (III)). We now present a simplified definition of cores.

Definition 11. A (simple) core for a $\operatorname{KB} \mathcal{K}=(\mathcal{T}, \mathcal{A})$ is an interpretation $\mathcal{I}_{c}=\left(\Delta^{\mathcal{I}_{c}}, \mathcal{I}_{c}\right)$, where

(c1) $\Delta^{\mathcal{I}_{c}}=\mathrm{N}_{\mathrm{l}}(\mathcal{K}), a^{\mathcal{I}_{c}}=a$ for all $a \in \Delta^{\mathcal{I}_{c}}$, and ${ }^{\mathcal{I}_{c}}$ is an interpretation function with

(c2) $\mathcal{I}_{c} \models \mathcal{A}$, and

(c3) $\mathcal{I}_{c} \models \alpha$ for each $\alpha \in \mathcal{T}$ of type (N1), (N3) and (N4), i.e. for all but existential inclusions in $\mathcal{T}$.

The constants from $\mathrm{N}_{\mathrm{I}}(\mathcal{K})$ are the fringe individuals of $\mathcal{I}_{c}$.

Using the above definition instead of Definition 7 and assuming the absence of closed predicates, all proofs of Section 5 hold as is. It thus remains to appropriately modify the program constructed in Section 6.2 to accommodate the simplified definition of cores. As before, given a TBox $\mathcal{T}$, for each $A \in \mathrm{N}_{\mathrm{C}}(\mathcal{T})$, we use a fresh unary predicate $\bar{A}$, and for each $p \in \mathrm{N}_{\mathrm{R}}(\mathcal{T})$, a binary $\bar{p}$. We use $\hat{r}(x, y)$ to denote $r(x, y)$, if $r \in \mathrm{N}_{\mathrm{R}}$, and $r(y, x)$ if $r^{-} \in \mathrm{N}_{\mathrm{R}}$. Then the rules in (II) and (III) are replaced by the following disjunctive rules:

$$
\begin{aligned}
A(x) \vee \bar{A}(x) & \leftarrow \operatorname{ind}(x) & & \text { for all } A \in \mathrm{N}_{\mathrm{C}}(\mathcal{T}), \\
p(x, y) \vee \bar{p}(x, y) & \leftarrow \operatorname{ind}(x), \operatorname{ind}(y) & & \text { for all } p \in \mathrm{N}_{\mathrm{R}}(\mathcal{T}) \\
& \leftarrow \operatorname{ind}(x), B_{1}(x), \ldots, B_{n}(x) & & \text { for all } B_{1} \sqcap \cdots \sqcap B_{n} \sqsubseteq \\
& \bar{B}_{n+1}(x), \ldots, \bar{B}_{k}(x) & & \sqsubseteq B_{n+1} \sqcup \cdots \sqcup B_{k} \in \mathcal{T} \\
A^{\prime}(y) & \leftarrow A(x), \hat{r}(x, y) & & \text { for all } A \sqsubseteq \forall r . A^{\prime} \in \mathcal{T} \\
\hat{s}(x, y) & \leftarrow \hat{r}(x, y) & & \text { for all } r \sqsubseteq s \in \mathcal{T}
\end{aligned}
$$


The rules in (VII) are replaced using the following rules. They mark every type that contains a nominal, but the type is not realized in the current core. Note that, for each core $\mathcal{I}_{c}$ and each nominal $\{a\}$, the only type containing $\{a\}$ that is realized in $\mathcal{I}_{c}$ is the actual type $\tau=$ type $\left(a, \mathcal{I}_{c}\right)$ of $a$, which is in fact stored in the atom hasType ${ }^{k}(a, \operatorname{vec}(\tau))$. For this reason, to mark the nominal types that are not realized, it suffices to simply mark every type $\tau$ with $\{a\} \in \tau$ and $\tau \neq \operatorname{type}\left(a, \mathcal{I}_{c}\right)$. We can achieve this by adding to $P_{M}$ the following rules for all $B \in \mathrm{N}_{\mathrm{C}}^{+}(\mathcal{T})$ and all nominals $\{a\} \in \mathrm{N}_{\mathrm{C}}^{+}(\mathcal{T})$ :

$$
\begin{aligned}
& \operatorname{Marked}(\vec{x}) \leftarrow \operatorname{Type}(\vec{x}),\{a\} \in \vec{x}, \operatorname{hasType}^{k}(a, \vec{y}), B \in \vec{x}, B \notin \vec{y} \\
& \operatorname{Marked}(\vec{x}) \leftarrow \operatorname{Type}(\vec{x}),\{a\} \in \vec{x}, \operatorname{hasType}^{k}(a, \vec{y}), B \notin \vec{x}, B \in \vec{y}
\end{aligned}
$$

Finally, since now fringe elements are exactly the constants of the input ABox, the rules in (IX) are replaced by a single constraint as follows:

$$
\leftarrow \operatorname{Marked}(\vec{x}), \text { RealizedType }(\vec{x})
$$

Note that in the absence of closed predicates, with the above rules replacing (II), (III), (VII) and (IX), our rewriting does not use not in rule bodies. Based on these observations, we obtain the following:

Theorem 5. For a c-acyclic OMQ $Q=(\mathcal{T}, \mathfrak{q})$, where $\mathcal{T}$ is an $\mathcal{A} \mathcal{L C H O I}$ TBox, we can build in polynomial time a query $\left(P_{Q}, q\right)$, where $P_{Q}$ is a positive disjunctive DATALOG program such that $\operatorname{cert}(Q, \mathcal{A})=$ $\operatorname{cert}\left(\left(P_{Q}, q\right), \mathcal{A}\right)$ for any given $A B o x \mathcal{A}$ over the concept and role names occurring in $\mathcal{T}$. In addition, if $\mathcal{T}$ is an $\mathcal{A L C H I}$ TBox, then $P_{Q}$ has no occurrences of the $\neq$ predicate.

We note that $\neq$-free positive disjunctive programs are not expressive enough to capture instance queries $Q=(\mathcal{T}, \mathfrak{q})$ when $\mathcal{T}$ has nominals. This follows from the following observation. For any positive $\neq$-free program $P$ and a set of facts $F$, if $P \cup F$ has a model, then also $P \cup F^{\prime}$ has a model, were $F^{\prime}$ is obtained from $F$ by renaming its constants with fresh ones that do not occur in $P \cup F$. However, this property cannot be recast to $\mathcal{A} \mathcal{L C H O \mathcal { I }}$. Take the TBox $\mathcal{T}=\{A \sqsubseteq\{a\}\}$ and observe that $\mathcal{T}$ is consistent with respect to the ABox $\mathcal{A}_{1}=\{A(a)\}$, but is inconsistent with respect to the $\mathrm{ABox} \mathcal{A}_{2}=\{A(b)\}$.

\section{The Need for Two Constants}

We note that the rewriting presented in Section 6.2 uses two distinct constants (namely 0 and 1), which are "introduced" by means of two facts that are always present in the constructed program. We observe that if no constants are allowed in rules, a polynomial time rewriting into a DATALOG program with negation or a positive disjunctive DATALOG program does not exist even for $\mathcal{A L C}$ TBoxes in the absence of closed predicates, under common assumptions in complexity theory. This can be argued using the well-known fact that deciding $(\{A(c)\}, \mathcal{T}) \models B(c)$, where $A, B$ are concept names and $\mathcal{T}$ is an $\mathcal{A L C}$ TBox, is an EXPTIME-hard problem. Assume a triple $A(c), B(c), \mathcal{T}$ as above, and suppose that from $\mathcal{T}$ we can compute in polynomial time a desired (polynomially sized) program $P_{\mathcal{T}}$ that does not use constants. Since $P_{\mathcal{T}}$ is a proper rewriting, it is the case that $(\{A(c)\}, \mathcal{T}) \models B(c)$ iff $B(c) \in J$ for every stable model $J$ of $P_{\mathcal{T}} \cup\{A(c)\}$. Since $A(c)$ is the only input fact, the grounding of $P_{\mathcal{T}} \cup\{A(c)\}$ is of polynomial size. From the complexity of DATALOG programs consisting of disjunctive positive rules, or consisting of non-disjunctive rules with negation under the stable model semantics, we obtain a coNP upper bound for testing $(\{A(c)\}, \mathcal{T}) \models B(c)$. This contradicts the belief that EXPTIME $\nsubseteq$ coNP.

\section{Conclusions and Future Work}

In this paper, we have proposed a novel technique for rewriting c-acyclic OMQs of the form $Q=(\mathcal{T}, \Sigma, \mathfrak{q})$, where $\mathcal{T}$ is an $\mathcal{A L C H O I}$ TBox, $\Sigma$ is a (possibly empty) set of closed predicates, and $\mathfrak{q}$ is a CQ, into a polynomially-sized DATALOG program $P_{Q}$ with negation under the stable model semantics. We have also shown that if $Q$ has no closed predicates (i.e., $\Sigma=\emptyset$ ) we can obtain a positive disjunctive DATALOG program 
with the built-in inequality predicate. If nominals are not present in the input TBox, the inequality predicate is unnecessary. To our best knowledge, these are the first such rewritings that take polynomial time to be computed.

Our rewriting establishes an interesting connection between two very prominent reasoning formalisms. On the one hand, the OMQs we consider allow for very rich ontological reasoning, and cover many of the most popular DL constructs (indeed, $\mathcal{A L C H O I}$ has most of the constructs present in $\mathcal{S H O I N}$, the basis of the OWL DL standard [32]). On the other hand, disjunctive DATALOG with negation as failure is a very prominent and versatile language for common-sense reasoning and problem solving. Our results show that the former can be effectively translated into the latter in polynomial time. To prove the correctness of this translation, we have used as an intermediate step a game-like characterization of the mentioned OMQs, which we believe is interesting in its own right. The fact that previous translations for similar languages required exponential time, points to the fact that, although related, these two formalisms can express knowledge in rather different ways. Given the differences in computational complexity of these formalisms, it is natural that the translation results in a program that is inherently non-ground, and uses predicates whose arity is not bounded. The presence of closed predicates makes our OMQs particularly well suited for settings in which complete and incomplete data coexist, allowing for non-monotonic inferences that exploit the knowledge about partial completeness. The price to pay for this is that we must target for the translation a variant of disjunctive DATALOG with negation. However, the use of negation as failure in the program resulting from our translation is rather limited.

Extensions and Future Work We have presented our results for $\mathcal{A L C H O I}$, but they also apply to $\mathcal{S H I O}$, using standard techniques to eliminate transitivity axioms (see, e.g., [33]). Moreover, the results can be easily generalized to $D L$-safe rules of [41]. These queries are syntactically restricted to ensure that the relevant variable assignments only map into individuals of the input ABox. We remark that our results also apply to other OMQs that can be reduced in polynomial time to the OMQs considered in this paper. For instance, some restricted forms of navigational queries (like, for example, nested regular path queries with at least one existentially quantified variable) can be reduced to our OMQs by adding a polynomial number of inclusions to the TBox, see for example [11].

Under common assumptions in complexity theory, our translation cannot be generalized to CQs, while remaining polynomial. This is because query answering for a disjunctive program with negation is in coNEXPTIME $^{\mathrm{NP}}$, but OMQ answering is 2EXPTIME-hard already for the DLs $\mathcal{A L C} \mathcal{I}$ [37] or $\mathcal{A L C O}$ [42]. Adapting the ideas in this work to CQs may be possible at the cost of an exponential blow-up, but we believe it would be technically quite involved. An interesting task for future direction is to obtain a polynomial translation for $\mathcal{A L C H O I} \mathcal{Q}$, which adds number restrictions to $\mathcal{A L C H O I}$. Considering other query languages, such as variations of regular path queries [16], is also a compelling direction for future research.

\section{Acknowledgments}

This work was supported by the Austrian Science Fund (FWF) projects P30360, P30873, and W1255-N23.

\section{References}

[1] Shqiponja Ahmetaj, Magdalena Ortiz, and Mantas Šimkus. Polynomial datalog rewritings for expressive description logics with closed predicates. In IJCAI, pages 878-885. IJCAI/AAAI Press, 2016.

[2] Shqiponja Ahmetaj, Magdalena Ortiz, and Mantas Šimkus. Polynomial disjunctive datalog rewritings of instance queries in expressive description logics. In Proc. of DL 2016, 2016.

[3] Shqiponja Ahmetaj, Magdalena Ortiz, and Mantas Šimkus. Rewriting guarded existential rules into small datalog programs. In ICDT, volume 98 of LIPICS, pages 4:1-4:24. Schloss Dagstuhl - LeibnizZentrum fuer Informatik, 2018. 
[4] Franz Baader, Diego Calvanese, Deborah McGuinness, Daniele Nardi, and Peter Patel-Schneider, editors. The Description Logic Handbook: Theory, Implementation, and Applications. Cambridge University Press, second edition, 2007.

[5] Labinot Bajraktari, Magdalena Ortiz, and Mantas Simkus. Combining rules and ontologies into clopen knowledge bases. In AAAI, pages 1728-1735, 2018. URL https://www.aaai.org/ocs/index.php/AAAI/AAAI18/paper/view/16991.

[6] Vince Bárány, Michael Benedikt, and Balder ten Cate. Rewriting guarded negation queries. In Proc. of MFCS' 13, pages 98-110. ACM, 2013.

[7] Michael Benedikt and Pierre Bourhis. Pspace hardness of mixed world query answering for atomic queries under guarded tgds. Technical report, University of Oxford, 2018. URL http://www.cs.ox.ac.uk/people/michael.benedikt/pspace.pdf

[8] Michael Benedikt, Pierre Bourhis, Balder ten Cate, and Gabriele Puppis. Querying visible and invisible information. In LICS, pages 297-306. ACM, 2016.

[9] Michael Benedikt, Bernardo Cuenca Grau, and Egor V. Kostylev. Source information disclosure in ontology-based data integration. In AAAI, pages 1056-1062. AAAI Press, 2017.

[10] Meghyn Bienvenu and Magdalena Ortiz. Ontology-mediated query answering with data-tractable description logics. In Reasoning Web, volume 9203 of Lecture Notes in Computer Science, pages 218-307. Springer, 2015.

[11] Meghyn Bienvenu, Diego Calvanese, Magdalena Ortiz, and Mantas Šimkus. Nested regular path queries in description logics. In KR. AAAI Press, 2014.

[12] Meghyn Bienvenu, Balder ten Cate, Carsten Lutz, and Frank Wolter. Ontology-based data access: A study through disjunctive datalog, csp, and MMSNP. ACM Trans. Database Syst., 39(4):33:1-33:44, 2014.

[13] Andrea Calì, Georg Gottlob, and Thomas Lukasiewicz. A general datalog-based framework for tractable query answering over ontologies. In PODS, pages 77-86. ACM, 2009.

[14] Diego Calvanese, Giuseppe De Giacomo, Domenico Lembo, Maurizio Lenzerini, and Riccardo Rosati. Tractable reasoning and efficient query answering in description logics: The DL-Lite family. J. Autom. Reasoning, 39(3):385-429, 2007.

[15] Diego Calvanese, Giuseppe De Giacomo, Domenico Lembo, Maurizio Lenzerini, and Riccardo Rosati. Data complexity of query answering in description logics. Artif. Intell., 195:335-360, 2013.

[16] Diego Calvanese, Thomas Eiter, and Magdalena Ortiz. Answering regular path queries in expressive description logics via alternating tree-automata. Inf. Comput., 237:12-55, 2014.

[17] Evgeny Dantsin, Thomas Eiter, Georg Gottlob, and Andrei Voronkov. Complexity and expressive power of logic programming. ACM Comput. Surv., 33(3):374-425, 2001.

[18] Ting Deng, Wenfei Fan, and Floris Geerts. Capturing missing tuples and missing values. ACM Trans. Database Syst., 41(2):10:1-10:47, 2016.

[19] Thomas Eiter, Georg Gottlob, and Heikki Mannila. Disjunctive datalog. ACM Trans. Database Syst., 22(3):364-418, 1997.

[20] Thomas Eiter, Giovambattista Ianni, Thomas Lukasiewicz, Roman Schindlauer, and Hans Tompits. Combining answer set programming with description logics for the semantic web. Artif. Intell., 172(12-13):1495-1539, 2008. doi: 10.1016/j.artint.2008.04.002. URL https://doi.org/10.1016/j.artint.2008.04.002. 
[21] Thomas Eiter, Magdalena Ortiz, Mantas Simkus, Trung-Kien Tran, and Guohui Xiao. Query rewriting for horn-shiq plus rules. In AAAI. AAAI Press, 2012.

[22] Thomas Eiter, Magdalena Ortiz, and Mantas Šimkus. Conjunctive query answering in the description logic SH using knots. J. Comput. Syst. Sci., 78(1):47-85, 2012.

[23] Wenfei Fan and Floris Geerts. Relative information completeness. ACM Trans. Database Syst., 35(4): 27:1-27:44, 2010.

[24] Enrico Franconi, Yazmin Angélica Ibáñez-García, and Inanç Seylan. Query answering with dboxes is hard. Electr. Notes Theor. Comput. Sci., 278:71-84, 2011.

[25] Michael Gelfond and Vladimir Lifschitz. The stable model semantics for logic programming. In Proc. of ICLP/SLP 1988. MIT Press, 1988.

[26] Georg Gottlob and Thomas Schwentick. Rewriting ontological queries into small nonrecursive datalog programs. In KR. AAAI Press, 2012.

[27] Georg Gottlob, Stanislav Kikot, Roman Kontchakov, Vladimir V. Podolskii, Thomas Schwentick, and Michael Zakharyaschev. The price of query rewriting in ontology-based data access. Artif. Intell., 213: 42-59, 2014.

[28] Georg Gottlob, Marco Manna, and Andreas Pieris. Polynomial combined rewritings for existential rules. In KR. AAAI Press, 2014.

[29] Georg Gottlob, Sebastian Rudolph, and Mantas Šimkus. Expressiveness of guarded existential rule languages. In PODS, pages 27-38. ACM, 2014.

[30] Georg Gottlob, Marco Manna, and Andreas Pieris. Polynomial rewritings for linear existential rules. In IJCAI, pages 2992-2998. AAAI Press, 2015.

[31] I. Horrocks, U. Sattler, S. Tessaris, and S. Tobies. Query containment using a DLR ABox. LTCS-Report LTCS-99-15, LuFG Theoretical Computer Science, RWTH Aachen, Germany, 1999. See http://wwwlti.informatik.rwth-aachen.de/Forschung/Reports.html.

[32] Ian Horrocks and Peter F. Patel-Schneider. KR and reasoning on the semantic web: OWL. In Handbook of Semantic Web Technologies, pages 365-398. Springer, 2011.

[33] Ullrich Hustadt, Boris Motik, and Ulrike Sattler. Reasoning in description logics by a reduction to disjunctive datalog. J. Autom. Reasoning, 39(3):351-384, 2007.

[34] Mark Kaminski, Yavor Nenov, and Bernardo Cuenca Grau. Datalog rewritability of disjunctive datalog programs and non-horn ontologies. Artif. Intell., 236:90-118, 2016.

[35] Roman Kontchakov, Carsten Lutz, David Toman, Frank Wolter, and Michael Zakharyaschev. The combined approach to ontology-based data access. In IJCAI, pages 2656-2661. IJCAI/AAAI, 2011.

[36] Thomas Lukasiewicz. A novel combination of answer set programming with description logics for the semantic web. IEEE Trans. Knowl. Data Eng., 22(11):1577-1592, 2010. doi: 10.1109/TKDE.2010.111.

[37] Carsten Lutz. The complexity of conjunctive query answering in expressive description logics. In Automated Reasoning, 4th International Joint Conference, IJCAR, pages 179-193, 2008.

[38] Carsten Lutz, Inanç Seylan, and Frank Wolter. Ontology-based data access with closed predicates is inherently intractable(sometimes). In IJCAI, pages 1024-1030. IJCAI/AAAI, 2013.

[39] Carsten Lutz, Inanç Seylan, and Frank Wolter. Ontology-mediated queries with closed predicates. In IJCAI, pages 3120-3126. AAAI Press, 2015. 
[40] Boris Motik and Riccardo Rosati. Reconciling description logics and rules. J. ACM, 57(5):30:1-30:62, 2010. doi: 10.1145/1754399.1754403. URL https://doi.org/10.1145/1754399.1754403.

[41] Boris Motik, Ulrike Sattler, and Rudi Studer. Query answering for OWL-DL with rules. J. Web Sem., 3 (1):41-60, 2005.

[42] Nhung Ngo, Magdalena Ortiz, and Mantas Simkus. Closed predicates in description logics: Results on combined complexity. In KR, pages 237-246, 2016. URL http://www.aai.org/ocs/index.php/KR/KR16/paper/view/12906.

[43] Magdalena Ortiz, Sebastian Rudolph, and Mantas Šimkus. Worst-case optimal reasoning for the horn-dl fragments of OWL 1 and 2. In KR. AAAI Press, 2010.

[44] Héctor Pérez-Urbina, Boris Motik, and Ian Horrocks. Tractable query answering and rewriting under description logic constraints. J. Applied Logic, 8(2):186-209, 2010.

[45] Simon Razniewski and Werner Nutt. Databases under the partial closed-world assumption: A survey. In Grundlagen von Datenbanken, volume 1313 of CEUR Workshop Proceedings, pages 59-64, 2014.

[46] Riccardo Rosati. Dl+log: Tight integration of description logics and disjunctive datalog. In Patrick Doherty, John Mylopoulos, and Christopher A. Welty, editors, Proceedings, Tenth International Conference on Principles of Knowledge Representation and Reasoning, Lake District of the United Kingdom, June 2-5, 2006, pages 68-78. AAAI Press, 2006. ISBN 978-1-57735-271-6. URL http://www.aaai.org/Library/KR/2006/kr06-010.php

[47] Andrea Schaerf. Reasoning with individuals in concept languages. Data and Knowledge Engineering, 13(2):141-176, 1994. doi: 10.1016/0169-023X(94)90002-7.

[48] Klaus Schild. A correspondence theory for terminological logics: Preliminary report. pages 466-471, 1991.

[49] Inanç Seylan, Enrico Franconi, and Jos de Bruijn. Effective query rewriting with ontologies over DBoxes. In Proc. of IJCAI 2009, 2009.

[50] Frantisek Simancik, Yevgeny Kazakov, and Ian Horrocks. Consequence-based reasoning beyond horn ontologies. In IJCAI, pages 1093-1098. IJCAI/AAAI, 2011.

[51] Despoina Trivela, Giorgos Stoilos, Alexandros Chortaras, and Giorgos B. Stamou. Optimising resolution-based rewriting algorithms for OWL ontologies. J. Web Sem., 33:30-49, 2015.

[52] Mihalis Yannakakis. Algorithms for acyclic database schemes. In Proceedings of the Seventh International Conference on Very Large Data Bases - Volume 7, VLDB '81, pages 82-94. VLDB Endowment, 1981. URL http://dl.acm.org/citation.cfm?id=1286831.1286840 


\section{Appendix}

Proof of Proposition 2 We provide the missing, more technical, part of the proof of Proposition 2 , We have already shown that $\mathcal{J}$ is an extension of $\mathcal{I}_{c}$ and that $\mathcal{J} \models_{\Sigma} \mathcal{A}$. To complete the proof of soundness, it is left to show that $\mathcal{J} \models \mathcal{T}$, more precisely that $\mathcal{J}$ satisfies all inclusions of the forms (N1), (N2), (N3), and $(\mathrm{N} 4)$ in $\mathcal{T}$. We show the claim for each such inclusion next.

(N1) For inclusions of the form $B_{1} \sqcap \cdots \sqcap B_{n} \sqsubseteq B_{n+1} \sqcup \cdots \sqcup B_{k}$, by construction of $\mathcal{J}$ and by definition of non-losing str, for all $w \in \Delta^{\mathcal{J}}$, type $(w, \mathcal{J})=\operatorname{tail}(w)$ and $\operatorname{tail}(w) \in \operatorname{LC}\left(\mathcal{T}, \Sigma, \mathcal{I}_{c}\right)$ - that is, $\operatorname{tail}(w)$ satisfies all inclusions of type (N1). Hence $\mathcal{J}$ satisfies all inclusions of type (N1).

(N2) Consider an inclusion $\alpha=A \sqsubseteq \exists r . A^{\prime} \in \mathcal{T}$. We distinguish the following cases:

- If $r \in_{\mathcal{T}} \Sigma$, that is there is some inclusion $r \sqsubseteq_{\mathcal{T}}^{*} s$ with $s^{(-)} \in \Sigma$, then that $\mathcal{J}$ satisfies $\alpha$ is a direct consequence of the definition of a core, more precisely that $\mathcal{I}_{c} \models A \sqsubseteq \exists r . A^{\prime}$, and the fact that $\mathcal{J}$ is an extension of $\mathcal{I}_{c}$.

- If $r \notin \mathcal{T} \quad \Sigma$, let $w$ be an arbitrary object in $A^{\mathcal{J}}$. We show that there exists a $v \in \Delta^{\mathcal{J}}$ such that $(w, v) \in r^{\mathcal{J}}$ and $v \in A^{\prime \mathcal{J}}$. By construction of $A^{\mathcal{J}}$ and by construction of $\operatorname{frn}\left(\mathcal{I}_{c}, \operatorname{str}\right), A \in \operatorname{tail}(w)$ holds and $w$ is a run that follows str. Since $\operatorname{str}$ is a non-losing strategy $\operatorname{str}(\operatorname{tail}(w), \alpha)$ is defined, that is there exists a $\tau^{\prime}$ over $\mathcal{T}$ such that $\operatorname{str}(\operatorname{tail}(w), \alpha)=\tau^{\prime}$ and $A^{\prime} \in \tau^{\prime}$. We further distinguish the following cases:

- If $w=a$ for an individual $a \in \Delta^{\mathcal{I}_{c}}$ and $\tau^{\prime}$ is a c-type, then the assumption holds by definition of a strategy, that is there exists $\left(a, a^{\prime}\right) \in r^{\mathcal{I}_{c}}$ with $\tau^{\prime}=\left(a^{\prime}, \mathcal{I}_{c}\right)$, and thus also $a^{\prime} \in A^{\prime \mathcal{J}}$.

- Otherwise, if $w=a$ and $\tau^{\prime}$ is not a c-type, then we have $v=a \alpha \tau^{\prime} \in \operatorname{frn}\left(\mathcal{I}_{c}, s t r\right)=\Delta^{\mathcal{J}}$, and by construction either $(a, v) \in r^{\mathcal{J}}$ if $r$ is a role name, or $(v, a) \in\left(r^{-}\right)^{\mathcal{J}}$ if $r^{-}$is a role name. Since $A^{\prime} \in \operatorname{tail}\left(a \alpha \tau^{\prime}\right)$, then $a \alpha \tau^{\prime} \in A^{\prime \mathcal{J}}$ as desired.

- The case when $w$ is a run of the form $a \ldots \alpha_{\ell} \tau_{\ell}$ and $\tau^{\prime}$ is not a c-type is similar, that is, $w \alpha \tau^{\prime} \in \operatorname{frn}\left(\mathcal{I}_{c}, s t r\right)=\Delta^{\mathcal{J}}$, and as $A^{\prime} \in \tau^{\prime}$, we have $w \alpha \tau^{\prime} \in A^{\prime \mathcal{J}}$, and either $\left(w, w \alpha \tau^{\prime}\right) \in$ $r^{\mathcal{J}}$ if $r$ is a role name, or $\left(w \alpha \tau^{\prime}, w\right) \in\left(r^{-}\right)^{\mathcal{J}}$ if $r^{-}$is a role name.

- Finally, assume $w$ is a run of the form $a \ldots \alpha_{\ell} \tau_{\ell}$ and $\tau^{\prime}$ is a c-type. It follows that $v=$ $w \alpha \tau^{\prime} \in \operatorname{crn}\left(\mathcal{I}_{c}, s t r\right)$, and hence by construction, $\left(w, a_{\tau^{\prime}}\right) \in r^{\mathcal{J}}$ if $r$ is a role name, or $\left(a_{\tau^{\prime}}, w\right) \in\left(r^{-}\right)^{\mathcal{J}}$ if $r^{-}$is a role name, where $a_{\tau^{\prime}}$ is the chosen individual that realizes $\tau^{\prime}$ in $\mathcal{I}_{c}$. Clearly, since $A^{\prime} \in \tau^{\prime}$, we have $a_{\tau^{\prime}} \in A^{\prime \mathcal{J}}$.

(N3) Consider an inclusion $\alpha=A_{1} \sqsubseteq \forall r . A_{2}$. To show $\mathcal{J} \models \alpha$, let $w$ be an arbitrary object in $A_{1}^{\mathcal{J}}$. By construction of $\mathcal{J}, w \in A_{1}^{\mathcal{J}}$ implies $A_{1} \in \operatorname{tail}(w)$. Consider an arbitrary object $w^{\prime} \in \Delta^{\mathcal{J}}$ such that $\left(w, w^{\prime}\right) \in r^{\mathcal{J}}$; note that the claim trivially holds for $w$ in case there is no such $w^{\prime}$. To show that $w^{\prime} \in A_{2}^{\mathcal{J}}$, we distinguish the following cases.

- Both $w$ and $w^{\prime}$ are individuals from the core, namely $a$ and $a^{\prime}$, respectively. Since $\mathcal{J}$ is an extension of $\mathcal{I}_{c}$, then $\left(a, a^{\prime}\right) \in r^{\mathcal{I}_{c}}$. By definition of a core, $\mathcal{I}_{c} \models A_{1} \sqsubseteq \forall r . A_{2}$ and, therefore, $a^{\prime} \in A_{2}^{\mathcal{I}_{c}}$. Since $\mathcal{J}$ is an extension of $\mathcal{I}_{c}$, we have $a^{\prime} \in A_{2}^{\mathcal{J}}$.

If at least $w$ or $w^{\prime}$ is not an individual, then, by the definition of $r^{\mathcal{J}}$, we have four possible cases:

- $w$ is arbitrary, and $w^{\prime}$ is of the form $w \alpha_{i} \tau_{i}$ for some inclusion $\alpha_{i}=A \sqsubseteq \exists r_{i} . A^{\prime} \in \mathcal{T}$ with $r_{i} \notin_{\mathcal{T}} \Sigma$. We further distinguish two cases.

- If $r$ is a role name, then, by construction of $\mathcal{J}, r_{i} \sqsubseteq_{\mathcal{T}}^{*} r$ follows. As $w \alpha_{i} \tau_{i}$ is a run that follows str, $\tau_{i}$ must satisfy (C2). This together with the assumption $A_{1} \in \operatorname{tail}(w)$ and the fact that $r_{i} \sqsubseteq_{\mathcal{T}}^{*} r$, imply that $A_{2} \in \tau_{i}$, and $w^{\prime}=w \alpha_{i} \tau_{i} \in A_{2}^{\mathcal{J}}$ follows. 
- If $r^{-}$is a role name, then $\left(w \alpha_{i} \tau_{i}, w\right) \in\left(r^{-}\right)^{\mathcal{J}}$. By construction of $\mathcal{J}$, it must be the case that $r_{i}^{-} \sqsubseteq_{\mathcal{T}}^{*} r^{-}$, so $r_{i} \sqsubseteq_{\mathcal{T}}^{*} r$ follows, and we can argue as above, using (C2) to conclude that $A_{2} \in \tau_{i}$ and $w^{\prime}=w \alpha_{i} \tau_{i} \in A_{2}^{\mathcal{J}}$.

- $w^{\prime}$ is an arbitrary word, and $w$ is of the form $w^{\prime} \alpha_{i} \tau_{i} \in \Delta^{\mathcal{J}}$, for $\alpha_{i}$ an inclusion $A \sqsubseteq \exists r_{i} . A^{\prime} \in \mathcal{T}$ with $r_{i} \notin_{T} \Sigma$. We again distinguish two cases.

- If $r$ is a role name, then by construction of $\mathcal{J}, r_{i}^{-} \sqsubseteq_{\mathcal{T}}^{*} r$. As $w^{\prime} \alpha_{i} \tau_{i}$ is a run that follows str, then $A \in \operatorname{tail}\left(w^{\prime}\right), A^{\prime} \in \tau_{i}$, and $\tau_{i}$ must satisfy (C2). From $A_{1} \in \operatorname{tail}(w)$ and $\operatorname{tail}(w)=$ $\operatorname{tail}\left(w^{\prime} \alpha_{i} \tau_{i}\right)$ follows that $A_{1} \in \tau_{i}$. The latter together with $A^{\prime} \in \tau_{i}, A \in \operatorname{tail}\left(w^{\prime}\right)$, and $r_{i}^{-} \sqsubseteq_{\mathcal{T}}^{*} r$ imply that $A_{2} \in \operatorname{tail}\left(w^{\prime}\right)$, so $w^{\prime} \in A_{2}^{\mathcal{J}}$.

- If $r^{-}$is a role name, then $\left(w^{\prime}, w^{\prime} \alpha_{i} \tau_{i}\right) \in\left(r^{-}\right)^{\mathcal{J}}$. By construction of $\mathcal{J}, r_{i} \sqsubseteq_{\mathcal{T}}^{*} r^{-}$, so $r_{i}^{-} \sqsubseteq_{\mathcal{T}}^{*} r$ holds, and we can argue as above that $w^{\prime}$ in $A_{2}^{\mathcal{J}}$.

- $w$ is an arbitrary word other than an individual, and $w^{\prime}$ is an individual $a_{\tau_{i}}$, where $w \alpha_{i} \tau_{i} \in$ $\operatorname{crn}\left(\mathcal{I}_{c}, s t r\right)$ for some $\alpha_{i}=A \sqsubseteq \exists r_{i} . A^{\prime}$ with $r_{i} \notin_{T} \Sigma$, and $a_{\tau_{i}}$ realizes $\tau_{i}$ in $\mathcal{I}_{c}$. We again have two cases.

- If $r$ is a role name, then $r_{i} \sqsubseteq_{\mathcal{T}}^{*} r$. Since $w \alpha_{i} \tau_{i}$ is a run that follows $\operatorname{str}, A \in \operatorname{tail}(w), A^{\prime} \in \tau_{i}$, and $\tau_{i}$ must satisfy (C2). The latter together with $A_{1} \in \operatorname{tail}(w)$ and $r_{i} \sqsubseteq_{\mathcal{T}}^{*} r$ imply that $A_{2} \in \tau_{i}$. As $\tau_{i}=\operatorname{tail}\left(a_{\tau_{i}}\right)$, we have that $A_{2} \in \operatorname{tail}\left(a_{\tau_{i}}\right)$ and $a_{\tau_{i}} \in A_{2}^{\mathcal{J}}$.

- If $r^{-}$is a role name, then it is the case that $r_{i}^{-} \sqsubseteq_{\mathcal{T}}^{*} r^{-}$and $r_{i} \sqsubseteq_{\mathcal{T}}^{*} r$, so we argue as above that $w^{\prime}=a_{\tau_{i}} \in A_{2}^{\mathcal{J}}$.

- $w$ is an individual $a_{\tau_{i}}$, while $w^{\prime}$ is not an individual, and $w^{\prime} \alpha_{i} \tau_{i} \in \operatorname{crn}\left(\mathcal{I}_{c}, \operatorname{str}\right)$ for some $\alpha_{i}=$ $A \sqsubseteq \exists r_{i} . A^{\prime}$ with $r_{i} \notin \mathcal{T} \Sigma$, and $a_{\tau_{i}}$ realizes $\tau_{i}$ in $\mathcal{I}_{c}$. We again consider two cases.

- If $r$ is a role name, then $r_{i}^{-} \sqsubseteq_{\mathcal{T}}^{*} r$. As $w=a_{\tau_{i}} \in A_{1}^{\mathcal{J}}$ and $a_{\tau_{i}}$ is a run that follows str, by construction of $\mathcal{J}$ we have $A_{1} \in \operatorname{tail}\left(a_{\tau_{i}}\right)=\tau_{i}$. As $w^{\prime} \alpha_{i} \tau_{i}$ is a run that follows str, $A \in \operatorname{tail}\left(w^{\prime}\right), A^{\prime} \in \tau_{i}$, and $\tau_{i}$ must satisfy (C2). The latter together with $A_{1} \in \tau_{i}$ and $r_{i}^{-} \sqsubseteq r$ imply that $A_{2} \in \operatorname{tail}\left(w^{\prime}\right)$, hence $w^{\prime} \in A_{2}^{\mathcal{J}}$.

- If $r^{-}$is a role name, then it is the case that $r_{i}^{-} \sqsubseteq_{\mathcal{T}}^{*} r^{-}$and $r_{i} \sqsubseteq_{\mathcal{T}}^{*} r$, and therefore we argue as above that $w^{\prime} \in A_{2}^{\mathcal{J}}$.

(N4) Finally, consider an inclusion $\alpha=r \sqsubseteq s \in \mathcal{T}$. For pairs of individuals, $\left(a, a^{\prime}\right) \in r^{\mathcal{J}}$ implies $\left(a, a^{\prime}\right) \in s^{\mathcal{J}}$ because $\mathcal{J}$ is an extension of $\mathcal{I}_{c}$ and $\mathcal{I}_{c} \models r \sqsubseteq s$. For all other pairs of objects, it is not hard to verify that $\mathcal{J} \models r \sqsubseteq s$ is guaranteed by the construction of $\mathcal{J}$, in particular the fact that $p^{\mathcal{J}}$ is closed under the role inclusions in $\mathcal{T}$, and the fact that, Otherwise, assume $(w, v) \in r^{\mathcal{J}}$, for an arbitrary pair of an object and its child, where at least one is not an individual. If $s$ is a role name $p$, then that $(w, v)$ belongs to $p^{\mathcal{J}}$ is ensured by construction of $\mathcal{J}$; otherwise if $r \sqsubseteq p^{-}$is in $\mathcal{T}$ for a role name $p$, then $r^{-} \sqsubseteq p$ is also in $\mathcal{T}$. 